\title{
Simulation Estimation of Dynamic Switching Regression and Dynamic Disequilibrium Models - Some Monte Carlo Results
}

\author{
Lung-fei Lee* \\ Department of Economics \\ The Hong Kong University of Science and Technology \\ Clear Water Bay, Kowloon, Hong Kong \\ and \\ Department of Economics \\ The University of Michigan \\ Ann Arbor, Michigan, USA \\ First draft: dated January 30, 1995 \\ Second revision: dated October 9, 1995 \\ Final revision dated
}

March 19, 1996

\begin{abstract}
This article considers the estimation of dynamic exogenous switching regression models and dynamic endogenous switching models. With autocorrelation in disturbances or latent lagged dependent variables, likelihood functions of such models involve high dimensional integrals and a huge number of summations over unobserved regime paths. Simulated likelihood methods and simulated methods of moments are available. These approaches simulate both continuous and discrete latent dependent variables. By Monte Carlo experiments, it is found that the performances of various approaches depend crucially on how discrete state variables are simulated. The valuable approach is to simulate regime paths with regime probabilities based on the current and past sample information.
\end{abstract}

Key words: Simulated likelihood; Regime classification; Dynamic switching model; Markov switching model; Disequilibrium model

JEL classification: $\mathrm{C} 15 ; \mathrm{C} 32$

\footnotetext{
*We would like to thank Professor G. Laroque and anonymous referees for their valuable comments and suggestions. Dr. Songnian Chen has provided helpful comments on an earlier version of this article and pointed out an important reference on this subject. This research has been supported by a DAG research grant from the Hong Kong U. of Science and Technology. An earlier version of this article was presented in the Econometric Society 7th World Congress in Tokyo, Japan, August 1995.

CORRESPONDENCE ADDRESS: Lung-fei Lee, Department of Economics, The Hong Kong University of Science and Technology, Clear Water Bay, Kowloon, Hong Kong. Tel: (852) 2358-7600; Fax: (852) 2358-2084; E-mail: Iflee@usthk.ust.hk
} 


\section{Introduction}

This article investigates econometric methods for the estimation of dynamic switching regression models and dynamic disequilibrium models. Earlier contributions on these models are the articles by Quandt (1974), Quandt and Ramsey (1978), Goldfeld and Quandt (1973), Fair and Jaffee (1972), Maddala and Nelson (1974), Goldfeld and Quandt (1975), and Kiefer (1978) among others. These models are used for the analysis of time series data. Due to computational complexity and the lack of computationally tractable statistical methods, those models have mostly been specified and estimated without dynamic structures. However, recent developments on simulation estimation methodologies may overcome such difficulties. It is the objective of this article to investigate possibly computationally tractable simulation methods for the estimation of such dynamic models.

Goldfeld and Quandt (1973) formulated switching regression models with dynamic structures where the regime switching process was modeled by a Markov chain. Under the assumption of no lagged latent dependent variables in regression equations, Cosslett and Lee (1985) suggested a recursive algorithm that is computationally tractable for the evaluation of the likelihood function. Subsequently, Hamilton (1989) introduced a filter algorithm (see also Kim 1994). The recursive algorithms in Cosslett and Lee and Hamilton provide efficient estimation and inference with the likelihood function. For more complicated models with lagged latent dependent variables, Cosslett and Lee have pointed out that the recursive algorithm originated in their article will not be applicable. So is the filtering algorithm in Hamilton (1989). When either serially correlated disturbances or lagged latent dependent variables appear in any switching regression equation of a model with $m$ regimes and $T$ periods, likelihood functions for such models involve $T$-fold integrals in addition to $m^{T}$-summations of regime paths. These likelihood functions are neither analytically nor computationally tractable with conventional quadrature methods.

Recent developments of simulation methods provide alternative approaches for handling multiple integrals (McFadden 1989; Pakes and Pollard 1989; Hajivassiliou and McFadden 1990; Borsch-Supan and Hajivassiliou 1993; Keane 1994, among others). For the static disequilibrium model, methods of simulated moments and simulated pseudo-likelihoods have been considered in Laroque and Salanie (1994). For dynamic disequilibrium models, simulated pseudo-likelihood methods have been proposed in Laroque and Salanie (1993) and methods for evaluating likelihood functions have been suggested in Hendry and Richard (1992). Lee (1994) has proposed a simulated likelihood approach for dynamic disequilibrium 
models where only an own lagged dependent variable of first order can appear in each switching regression equation. Based on a 'renewal' property of such models, only latent continuous dependent variables but not regime paths need be simulated and the simulated likelihood is smooth. While the latter models are quite important (Quandt 1988), general models that allow higher order latent lagged variables and serially correlated disturbances do not possess the 'renewal' property. For the estimation of general models, only the simulated pseudo-likelihood approaches of Laroque and Salanie and simulated likelihood approaches of Hendry and Richard remain to be feasible, at least, in principle. Other simulation approaches are also available for the estimation of stochastic variance models, dynamic stochastic volatility models, and various nonlinear time series models (Shephard 1993, Danielsson and Richard 1993, Smith 1993, Gourieroux et al. 1993, and others in a special issue on simulation techniques published by Journal of Applied Econometrics).

This article follows up the simulation estimation literature to investigate potentially computationally tractable simulated likelihood methods for the estimation of dynamic switching regression and disequilibrium models. We report our investigation and findings based on Monte Carlo experiments. Various possible approaches are compared in terms of computational tractability, finite sample performance of simulated estimators, and regime classification. ${ }^{1}$

The organization of this article is as follows. In Section 2, we review some dynamic models. Likelihood functions for such models are derived. Several simulation algorithms are described in Section 3. Regime classification rules derived from simulated likelihood functions are suggested in Section 4. Monte Carlo experiments for a dynamic switching regression model are reported in Section 5. Section 6 reports Monte Carlo results for a dynamic disequilibrium model. We summarize our findings in Section 7.

\section{Exogenous and Endogenous Switching Regression Models}

Goldfeld and Quandt (1973) considered a situation in which observations, indexed by $t, t=1, \ldots, T$, are available on a dependent variable $y_{t}$ and on a finite vector of independent variables $x_{t}$. The $y_{t}$ is assumed to be generated by one of the $m$ regression equations. The simplest model is specified when the probabilities of switching are independent of what state the system was in in the previous period and there are no serial correlations in regression equations. Estimation by the method of maximum likelihood

\footnotetext{
${ }^{1}$ Hendry and Richard (1992) provided Monte Carlo evidences on the accuracy of a simulated likelihood function for a simple one parameter dynamic disequilibrium model. They evaluated the case of a time series with 50 periods based on 10,000 Monte Carlo drawings. They have investigated neither simulated likelihood estimators nor the issue of regime classsification.
} 
and methods of moments has been considered in Quandt and Ramsey (1978). By relaxing the simple switching mechanism, a Markov switching model was introduced in Goldfeld and Quandt (1973). Let $I_{t}$ be the (latent) regime indicator at $t$, i.e., $I_{t}=i$ if the state is $i$ at period $t$. By introducing a transition matrix $\Lambda$, the regime switching process is a Markov chain:

$$
\left[P\left(I_{t-1}=1\right), \ldots, P\left(I_{t-1}=m\right)\right] \Lambda=\left[P\left(I_{t}=1\right), \ldots, P\left(I_{t}=m\right)\right]
$$

where $P\left(I_{t}=i\right)$ is the probability in state $i$ at $t$. Under these specifications and with unknown parameters suppressed, the log likelihood function is

$$
L=\ln \left\{\sum_{I_{T}=1}^{m} \cdots \sum_{I_{1}=1}^{m}\left[\prod_{t=1}^{T} f\left(y_{t} \mid I_{t}\right) \cdot \prod_{t=2}^{T} P\left(I_{t} \mid I_{t-1}\right) \cdot P\left(I_{1}\right)\right]\right\},
$$

where $P\left(I_{t} \mid I_{t-1}\right)$ is the transition probability from state $I_{t-1}$ at time $t-1$ to state $I_{t}$ at time $t$ and $f\left(y_{t} \mid I_{t}=i\right)$ is the probability density of $y_{t}$ from the regression equation of regime $i$ (Cosslett and Lee 1985). Direct evaluation of the likelihood function of (2) involves the sum of $m^{T}$ terms and is computationally intractable except for small $T$. This difficulty, however, can be overcome by a recurrence algorithm in Cosslett and Lee (1985) and a filtering algorithm in Hamilton (1989).

As it was noted in Goldfeld and Quandt (1973), error terms in regression equations might likely be autocorrelated. Denote the dependent variable in regime $i$ at time $t$ by $y_{i t}^{*}$ with a superscript $*$ to distinguish it from the observed $y_{t}$. The observed $y_{t}$ relates to the underlying regression equations by $y_{t}=y_{i t}^{*}$ if $I_{t}=i$. A first-order Markov process, $u_{i t}=\rho_{i} u_{i, t-1}+\epsilon_{i t}$, will imply that $y_{i t}^{*}=\rho_{i} y_{i, t-1}^{*}+\left(x_{t}-\rho_{i} x_{t-1}\right) \beta_{i}+\epsilon_{i t}$. For dynamic models, one may introduce both latent and observed lagged dependent variables in switching equations. At each $t$, only one of $y_{i t}^{*}, i=1, \cdots, m$, can be observed and the remaining ones are not. Corresponding to the value of $I_{t}$, let $z_{t}^{*}$ be the $(m-1)$-dimensional subvector consisting of unobservable dependent variables. Let $\bar{y}_{t}=\left(y_{1}, \cdots, y_{t}\right), \bar{I}_{t}=\left(I_{1}, \cdots, I_{t}\right)$, and $\bar{z}_{t}^{*}=\left(z_{1}^{*}, \cdots, z_{t}^{*}\right)$ for simplicity. Let $h\left(\bar{y}_{T}, \bar{I}_{T}, \bar{z}_{T}^{*}\right)$ be the joint density of $\bar{y}_{T}, \bar{I}_{T}$ and $\bar{z}_{T}^{*}$. The likelihood function is

$$
h\left(\bar{y}_{T}\right)=\sum_{I_{T}=1}^{m} \cdots \sum_{I_{1}=1}^{m} \int_{-\infty}^{\infty} \cdots \int_{-\infty}^{\infty} h\left(\bar{y}_{T}, \bar{I}_{T}, \bar{z}_{T}^{*}\right) d z_{1}^{*} \cdots d z_{T}^{*} .
$$

The $T$ summations sum up all the likelihood components over the $m^{T}$ regime paths. The integrals are integrated over all latent continuous dependent variables for each regime path. The recurrence algorithm in Cosslett and Lee (1983) and Familton's filter can not be applied because they rely on the Markov property of both regime switching and the observed dependent variables. The Markov property does not 
hold in the presence of latent lagged dependent variables. For such dynamic models, one has to face both the $m^{T}$-summation problem and the high dimensional integrals problem.

The formulation of regime switching in the above models is known as exogenous switching. There are endogenous switching models where the regime switching mechanism involves dependent variables of regression equations. An important class of endogenous switching models consists of disequilibrium models. Quandt (1988, p.134) formulated a dynamic disequilibrium model of the form: $y_{1 t}^{*}=x_{1 t} \beta_{1}+$ $\lambda_{1}\left(y_{1, t-1}^{*}-y_{t-1}\right)+\epsilon_{1 t}, y_{2 t}^{*}=x_{2 t} \beta_{2}+\lambda_{2}\left(y_{2, t-1}^{*}-y_{t-1}\right)+\epsilon_{2 t}$, and $y_{t}=\min \left\{y_{1 t}^{*}, y_{2 t}^{*}\right\}$. This model captures dynamic adjustments of unsatisfied demand or supply over time. The regime switching process is endogenously determined as $I_{t}=1$ if and only if $y_{2 t}^{*}>y_{1 t}^{*}$. For this model, the auxiliary latent variable $z_{t}^{*}$ is that $z_{t}^{*}=y_{2 t}^{*}$ if $I_{t}=1$ and $z_{t}^{*}=y_{1 t}^{*}$ if $I_{t}=2$. This $z_{t}^{*}$ represents the unobserved quantity (the long side of the market) at $t$. The likelihood function is

$$
h\left(\bar{y}_{T}\right)=\sum_{I_{T}=1}^{2} \cdots \sum_{I_{1}=1}^{2} \int_{y_{T}}^{\infty} \cdots \int_{y_{1}}^{\infty} h\left(\bar{y}_{T}, \bar{I}_{T}, \bar{z}_{T}^{*}\right) d z_{1}^{*} \cdots d z_{T}^{*} .
$$

Integrals in likelihood functions of endogenous switching models have usually restricted ranges. On the contrary, ranges of integrals for exogenous switching models are unrestricted. Detailed surveys on these models can be found in Quandt (1988).

\section{Simulation Estimation Approaches}

Consider first an exogenous dynamic switching model. The likelihood function has the general form in (3). We follow Hendry and Richard (1992) to consider simulation techniques based on factorizations of the sequential joint density of the observables and latent dependent variables. Let the past information set generated by $\bar{y}_{t-1}, \bar{I}_{t-1}$ and $\bar{z}_{t-1}^{*}$ be denoted by $\mathcal{J}_{t-1}$. A recurrence relation of special interest is

$$
h\left(\bar{y}_{t}, \bar{I}_{t}, \bar{z}_{t}^{*}\right)=g\left(z_{t}^{*} \mid I_{t}, y_{t}, \mathcal{J}_{t-1}\right) P\left(I_{t} \mid y_{t}, \mathcal{J}_{t-1}\right) f\left(y_{t} \mid \mathcal{J}_{t-1}\right) \cdot h\left(\bar{y}_{t-1}, \bar{I}_{t-1}, \bar{z}_{t-1}^{*}\right)
$$

The $g\left(z_{t}^{*} \mid I_{t}, y_{t}, \mathcal{J}_{t-1}\right)$ is the density of the unobserved dependent variables at $t$ given the past information and the knowledge which states have or have not been occupied at time $t$. The additional conditioning on $y_{t}$ is just to capture the possible contemporaneous correlation of $y_{t}$ with $z_{t}^{*}$. The second component is a conditional probability of regime occurrence given the past $\mathcal{J}_{t-1}$ and currently available sample $y_{t}$ at $t$. The $f\left(y_{t} \mid \mathcal{J}_{t-1}\right)$ is the density of the sample $y_{t}$ given the past $\mathcal{J}_{t-1}$. The last two components can be derived from a model specification of $P\left(I_{t} \mid \mathcal{J}_{t-1}\right)$ and $f\left(y_{t} \mid I_{t}=i, \mathcal{J}_{t-1}\right)$ where $i=1, \ldots, m$. It is obvious 
that

$$
f\left(y_{t} \mid \mathcal{J}_{t-1}\right)=\sum_{i=1}^{m} f\left(y_{t} \mid I_{t}=i, \mathcal{J}_{t-1}\right) P\left(I_{t}=i \mid \mathcal{J}_{t-1}\right)
$$

By the Bayes rule,

$$
P\left(I_{t} \mid y_{t}, \mathcal{J}_{t-1}\right)=f\left(y_{t} \mid I_{t}, \mathcal{J}_{t-1}\right) P\left(I_{t} \mid \mathcal{J}_{t-1}\right) / f\left(y_{t} \mid \mathcal{J}_{t-1}\right)
$$

Under the decomposition in (5), the likelihood function can be rewritten as

$$
\begin{aligned}
h\left(\bar{y}_{T}\right)= & \sum_{I_{1}=1}^{m} \cdots \sum_{I_{T}=1}^{m} \int_{-\infty}^{\infty} \cdots \int_{-\infty}^{\infty} \prod_{t=2}^{T} f\left(y_{t} \mid \mathcal{J}_{t-1}\right) g\left(z_{t}^{*} \mid I_{t}, y_{t}, \mathcal{J}_{t-1}\right) \\
& \cdot P\left(I_{t} \mid y_{t}, \mathcal{J}_{t-1}\right) \cdot f\left(y_{1}\right) g\left(z_{1}^{*} \mid I_{1}, y_{1}\right) P\left(I_{1} \mid y_{1}\right) \prod_{t=1}^{T} d z_{t}^{*}
\end{aligned}
$$

An alternative decomposition is

$$
h\left(\bar{y}_{t}, \bar{I}_{t}, \bar{z}_{t}^{*}\right)=g\left(z_{t}^{*} \mid I_{t}, y_{t}, \mathcal{J}_{t-1}\right) f\left(y_{t} \mid I_{t}, \mathcal{J}_{t-1}\right) P\left(I_{t} \mid \mathcal{J}_{t-1}\right) \cdot h\left(\bar{y}_{t-1}, \bar{I}_{t-1}, \bar{z}_{t-1}^{*}\right)
$$

With this recurrence formulae, the likelihood function can be rewritten as

$$
\begin{aligned}
h\left(\bar{y}_{T}\right)= & \sum_{I_{1}=1}^{m} \cdots \sum_{I_{T}=1}^{m} \int_{-\infty}^{\infty} \cdots \int_{-\infty}^{\infty} \prod_{t=2}^{T} f\left(y_{t} \mid I_{t}, \mathcal{J}_{t-1}\right) g\left(z_{t}^{*} \mid I_{t}, y_{t}, \mathcal{J}_{t-1}\right) \\
& \cdot P\left(I_{t} \mid \mathcal{J}_{t-1}\right) \cdot f\left(y_{1} \mid I_{1}\right) g\left(z_{1}^{*} \mid I_{1}, y_{1}\right) P\left(I_{1}\right) \prod_{t=1}^{T} d z_{t}^{*} .
\end{aligned}
$$

The decomposition in (9) seems natural as it translates the original model formulation in terms of density and probability functions. These recurrence relations and the corresponding likelihood functions of (8) and (10) motivate simulation algorithms for evaluating the likelihood function. The approaches will simulate both latent continuous dependent variables and discrete dependent variables.

At any possible value of the parameter vector, latent variables that need to be simulated at $t$ are $I_{t}$ and $z_{t}^{*}$. Since $z_{t}^{*}$ is defined conditional on $I_{t}$, it is imperative that $I_{t}$ be simulated first. Simulate $I_{t}$ from a multinomial distribution with probabilities $P\left(i \mid y_{t}, \mathcal{J}_{t-1}\right), i=1, \cdots, m$. Given a simulated $\mathcal{J}_{t-1}, P\left(i \mid y_{t}, \mathcal{J}_{t-1}\right)$ can be constructed from (6) and (7). Given simulated $I_{t}, z_{t}^{*}$ can be simulated from the density $g\left(z_{t}^{*} \mid I_{t}, y_{t}, \mathcal{J}_{t-1}\right)$. Given initial values, one simulates a path of regimes $\left(I_{1}, \cdots, I_{T}\right)$ and corresponding latent variables $\left(z_{1}^{*}, \cdots, z_{T}^{*}\right)$ for the whole sample periods. Such a simulation process can be independently repeated many times, say $S$. Let $I_{t}^{(s)}$ and $z_{t}^{*(s)}$ be, respectively, the simulated values of $I_{t}$ and $z_{t}^{*}$ at $t$ from the $s$ th simulation run. The likelihood function can then be simulated as

$$
h_{c}\left(\bar{y}_{T}\right)=\frac{1}{S} \sum_{s=1}^{S} \prod_{t=2}^{T} f\left(y_{t} \mid \mathcal{J}_{t-1}^{(s)}\right) \cdot f\left(y_{1}\right) .
$$


This simulated function is an unbiased estimate of the likelihood function $h\left(\bar{y}_{T}\right)$ given the sample observations. It is a consistent estimator of $h\left(\bar{y}_{T}\right)$ if $S$ goes to infinity. This is a simulated likelihood approach with simulated regime paths based on conditional sample probabilities (SLSP). This simulated likelihood function has an interesting property that it nests the likelihood function of the static model as a special case. In a static model, there are no latent lagged dependent variables in regression equations and the switching mechanism does not depend on previous states, i.e., $f\left(y_{t} \mid I_{t}, \mathcal{J}_{t-1}\right)=f\left(y_{t} \mid I_{t}\right)$ and $P\left(I_{t} \mid \mathcal{J}_{t-1}\right)=P\left(I_{t}\right)$. In this case, the simulated function $h_{c}\left(\bar{y}_{T}\right)$ reduces to $\prod_{t=1}^{T} f\left(y_{t}\right)$, which is exactly the likelihood for the classical static model.

For the likelihood in (10), $I_{t}$ can be simulated with transition probability $P\left(I_{t} \mid \mathcal{J}_{t-1}\right)$. With the simulated $I_{t}$ and $\mathcal{J}_{t-1}, z_{t}^{*}$ can be simulated with the density $g\left(z_{t}^{*} \mid I_{t}, y_{t}, \mathcal{J}_{t-1}\right)$. The likelihood function can be simulated as $h_{p}\left(\bar{y}_{T}\right)=\frac{1}{S} \sum_{s=1}^{S} \prod_{t=2}^{T} f\left(y_{t} \mid I_{t}^{(s)}, \mathcal{J}_{t-1}^{(s)}\right) \cdot f\left(y_{1} \mid I_{1}^{(s)}\right)$. This is a simulated likelihood with simulated regime paths based on transition probabilities. Based on the same likelihood function, there are other possible approaches. Instead of simulating $I_{t}$ based on $P\left(I_{t} \mid \mathcal{J}_{t-1}\right)$ (and $z_{t}^{*}$ based on $\left.g\left(z_{t}^{*} \mid I_{t}, \mathcal{J}_{t-1}\right)\right)$, it is possible to simulate $I_{t}$ randomly. Importance sampling is also possible. These other approaches do not, however, perform well in our subsequent Monte Carlo studies.

For dynamic disequilibrium models, regime probabilities are endogenously determined. The integrals of the likelihood function have limits determined by censoring due to the observed quantity being the minimum of supply and demand. For the $g\left(z_{t}^{*} \mid I_{t}, y_{t}, \mathcal{J}_{t-1}\right)$, the support of $z_{t}^{*}$ is $\left[y_{t}, \infty\right)$. Thus with the decomposition in (5), the likelihood function is

$$
\begin{aligned}
h\left(\bar{y}_{T}\right)= & \sum_{I_{1}=1}^{m} \cdots \sum_{I_{T}=1}^{m} \int_{y_{T}}^{\infty} \cdots \int_{y_{1}}^{\infty} \prod_{t=2}^{T} f\left(y_{t} \mid \mathcal{J}_{t-1}\right) g\left(z_{t}^{*} \mid I_{t}, y_{t}, \mathcal{J}_{t-1}\right) \\
& P\left(I_{t} \mid y_{t}, \mathcal{J}_{t-1}\right) \cdot f\left(y_{1}\right) g\left(z_{1}^{*} \mid I_{1}, y_{1}\right) P\left(I_{1} \mid y_{1}\right) \prod_{t=1}^{T} d z_{t}^{*} \\
= & \sum_{I_{1}=1}^{m} \cdots \sum_{I_{T}=1}^{m} \int_{-\infty}^{\infty} \cdots \int_{-\infty}^{\infty} \prod_{t=2}^{T} f\left(y_{t} \mid \mathcal{J}_{t-1}\right) g\left(z_{t}^{*} \mid I_{t}, y_{t}, \mathcal{J}_{t-1}\right) \\
& P\left(I_{t} \mid y_{t}, \mathcal{J}_{t-1}\right) \cdot f\left(y_{1}\right) g\left(z_{1}^{*} \mid I_{1}, y_{1}\right) P\left(I_{1} \mid y_{1}\right) \prod_{t=1}^{T} d z_{t}^{*} .
\end{aligned}
$$

The last expression in (12) does not have finite limits in its integrals as the limits are implicitly captured in the conditional density $g$. The simulation approaches described for the dynamic switching regression model are applicable to the dynamic disequilibrium model with the understanding that $z_{t}^{*}$ is simulated from a truncated density $g\left(z_{t}^{*} \mid I_{t}, y_{t}, \mathcal{J}_{t-1}\right)$ instead of an untruncated one. The simulation of truncated 
random variable is usually more complicated. This SLSP approach for evaluating the dynamic disequilibrium model has first appeared in Hendry and Richard (1992), p.11.

\section{Regime Classification}

As sample separation information is not available, one may like to identify the underlying regime that generates the sample observation at any sampling period. Such an issue is in the domain of discrimination analysis. A classification rule is optimal if it minimizes the total probability of missclassification (assuming parameters are known). At $t, y_{t}$ will be classified to regime $i$ if $P\left(I_{t}=i \mid \bar{y}_{T}\right)=\max \left\{P\left(I_{t}=j \mid \bar{y}_{T}\right): j=\right.$ $1, \cdots, m\}$. This classification rule is optimal (Anderson 1958). An optimal classification rule has been applied in Lee and Porter (1984) for a static switching regression model. For the Markov switching model, recursive smoothing algorithms have been proposed in Cosslett and Lee (1985), Hamilton (1989), and Kim (1994). For the Markov switching model, simulation is unnecessary for a smoothing algorithm. Simulation estimation is needed for a general dynamic switching model.

The probability $P\left(I_{t} \mid \bar{y}_{T}\right)$ is equal to $h\left(\bar{y}_{T}, I_{t}\right) / h\left(\bar{y}_{T}\right)$. For the dynamic switching regression model, $h\left(\bar{y}_{T}\right)$ is the likelihood function in (3) and

$$
h\left(\bar{y}_{T}, I_{t}\right)=\sum_{I_{1}=1}^{m} \cdots \sum_{I_{t-1}=1}^{m} \sum_{I_{t+1}=1}^{m} \cdots \sum_{I_{T}=1}^{m} \int_{-\infty}^{\infty} \cdots \int_{-\infty}^{\infty} h\left(\bar{y}_{T}, \bar{I}_{T}, \bar{z}_{T}^{*}\right) d z_{1}^{*} \cdots d z_{T}^{*} .
$$

For simulation, it is illustrative to rewrite (13) in a slightly different way. Define dichotomous indicators $D_{i}\left(I_{t}\right), i=1, \cdots, m$, such that $D_{i}\left(I_{t}\right)=1$ if $I_{t}=i ; 0$, otherwise. Then $h\left(\bar{y}_{T}, I_{t}=i\right)=h_{t i}\left(\bar{y}_{T}\right)$ where $h_{t i}\left(\bar{y}_{T}\right)=\sum_{I_{1}=1}^{m} \cdots \sum_{I_{T}=1}^{m} \int_{-\infty}^{\infty} \cdots \int_{-\infty}^{\infty} D_{i}\left(I_{t}\right) h\left(\bar{y}_{T}, \bar{I}_{T}, \bar{z}_{T}^{*}\right) d z_{1}^{*} \cdots d z_{T}^{*}$. For the dynamic disequilibrium model, the $h\left(\bar{y}_{T}\right)$ is in (4) and $h_{t i}\left(\bar{y}_{T}\right)=\sum_{I_{1}=1}^{m} \cdots \sum_{I_{T}=1}^{m} \int_{y_{T}}^{\infty} \cdots \int_{y_{1}}^{\infty} D_{i}\left(I_{t}\right) h\left(\bar{y}_{T}, \bar{I}_{T}, \bar{z}_{T}^{*}\right) d z_{1}^{*} \cdots d z_{T}^{*}$. These $h_{t i}\left(\bar{y}_{T}\right), i=1, \cdots, m$, can be simulated similarly to the simulation of likelihood functions. For the SLSP approach, $h_{t i}\left(\bar{y}_{T}\right)$ can be simulated as $h_{t i, c}\left(\bar{y}_{T}\right)$ where $h_{t i, c}\left(\bar{y}_{T}\right)=\frac{1}{S} \sum_{s=1}^{S} \prod_{r=1}^{T} D_{i}\left(I_{t}^{(s)}\right) f\left(y_{r} \mid \mathcal{J}_{r-1}^{(s)}\right)$ and $P\left(I_{t} \mid \bar{y}_{T}\right)$ can be simulated as $P_{c}\left(I_{t} \mid \bar{y}_{T}\right)$ where $P_{c}\left(I_{t} \mid \bar{y}_{T}\right)=h_{t i, c}\left(\bar{y}_{T}\right) / h_{c}\left(\bar{y}_{T}\right)$. The indicator $D_{i}\left(I_{t}^{(s)}\right)$ selects out simulated regime paths which go through the state $i$ at $t$. The simulator $P_{c}\left(I_{t} \mid \bar{y}_{T}\right)$ lies properly between zero and one. With unknown parameters substituted by simulated estimates, our suggested classification rule is to classify $y_{t}$ to regime $i$ if $P_{c}\left(I_{t}=i \mid \bar{y}_{T}\right)$ is the largest probability among the $m$ simulated conditional regime probabilities.

\section{Monte Carlo Studies on Dynamic Switching Regression Model}

For the dynamic model in the experiment, sample data are generated from a two-regimes dynamic 
model with the Markov switching:

$$
y_{1 t}^{*}=x_{1 t} \beta_{1}+\lambda_{1}\left(y_{1, t-1}^{*}-y_{t-1}\right)+\sigma_{1} \epsilon_{1 t},
$$

and

$$
y_{2 t}^{*}=x_{2 t} \beta_{2}+\lambda_{2}\left(y_{2, t-1}^{*}-y_{t-1}\right)+\sigma_{2} \epsilon_{2 t},
$$

where $\epsilon_{1 t}$ and $\epsilon_{2 t}$ are normally distributed as $N(0,1)$, independent of each other and serially uncorrelated. The $y_{1 t}^{*}$ and $y_{2 t}^{*}$ are, respectively, the latent dependent variables of regime 1 and regime 2. Regime occurrence is determined by a Markov Chain with two states as in (1). The transition matrix $\Lambda$ is a $2 \times 2$ matrix with transition probabilities being $p_{12}$ from regime 1 to regime 2 and $p_{21}$ from regime 2 to regime 1. The stationary regime probability for regime 1 is $p_{1} .=p_{21} /\left(p_{12}+p_{21}\right)$. The exogenous variables $x_{1 t}$ and $x_{2 t}$ are independently generated from a normal $N(0,2)$ random generator. The true parameters are $\beta_{1}=-1, \beta_{2}=1, \lambda_{1}=0.5, \lambda_{2}=0.5, \sigma_{1}=1$ and $\sigma_{2}=1$. The transition probabilities are $p_{12}=0.3$ and $p_{21}=0.3$. The stationary regime probabilities $p_{1}$. and $p_{2}$. are therefore 0.5 for each regime. The stochastic processes are running after 20 periods before sample observations are taken.

As the initial $y_{10}^{*}$ and $y_{20}^{*}$ are unobservable for the sample, an initial value problem exists for our estimation methods. In all the following Monte Carlo results, our strategy is to arbitrarily set the initial values $y_{10}^{*}=y_{20}^{*}=y_{0}(=0)$. This may not be an issue if sample periods are not too short. Non-negativity constraints for $\sigma_{1}, \sigma_{2}, p_{11}$ and $p_{22}$ are imposed by the following reparameterizations: $\sigma_{1}=\exp \left(\alpha_{1}\right)$, $\sigma_{2}=\exp \left(\alpha_{2}\right), p_{11}=1 /\left(1+\exp \left(\omega_{1}\right)\right)$ and $p_{22}=1 /\left(1+\exp \left(\omega_{2}\right)\right)$. As the magnitudes of $\lambda_{1}$ and $\lambda_{2}$ may theoretically be less than a unity (Quandt 1988), the transformations, $\lambda_{1}=\left(1-\exp \left(\gamma_{1}\right)\right) /\left(1+\exp \left(\gamma_{1}\right)\right)$ and $\lambda_{2}=\left(1-\exp \left(\gamma_{2}\right)\right) /\left(1+\exp \left(\gamma_{2}\right)\right)$, are also used. The parameters $\alpha_{1}, \alpha_{2}, \omega_{1}$, and $\omega_{2}$ are unconstrained.

\subsection{Simulated Likelihood Approaches}

For the SLSP approach, the simulated likelihood is $h_{c}$ in (11). For this particular two regimes model, the detailed steps are as follows. Throughout this section, $\phi(\cdot)$ and $\Phi(\cdot)$ denote, respectively, the standard normal density and probability functions.

Simulate a set of standard normal variables $\epsilon_{t}$ and a set of uniform random variables $u_{t}$ on $[0,1]$, $t=1, \ldots, T$, that are fixed and are invariant of parameters.

1. For period 1:

(a) Compute $R_{1}=M_{11}+M_{21}$ where $M_{11}=\frac{1}{\sigma_{1}} \phi\left(\frac{y_{1}-x_{11} \beta_{1}}{\sigma_{1}}\right) p_{1}$. and $M_{21}=\frac{1}{\sigma_{2}} \phi\left(\frac{y_{1}-x_{21} \beta_{2}}{\sigma_{2}}\right) p_{2}$. 
Simulate a regime indicator $I_{1}$ such that $I_{1}=1$ with probability $M_{11} /\left(M_{11}+M_{21}\right)$ using $u_{1}$, i.e., $I_{1}=1$ if $u_{1}<M_{11} /\left(M_{11}+M_{21}\right) ; I_{1}=2$, otherwise.

(b) Simulate $y_{1}^{*}=x_{21} \beta_{2}+\sigma_{2} \epsilon_{1}$ if $I_{1}=1$, but simulate $y_{1}^{*}=x_{11} \beta_{1}+\sigma_{1} \epsilon_{1}$ if $I_{1}=2$.

2. For period $t$ : The $I_{t-1}$ and $y_{t-1}^{*}$ have been simulated in the previous steps. $R_{t-1}$ has been evaluated. Define $y_{1, t-1}^{(p)}$ and $y_{2, t-1}^{(p)}$ such that $y_{1, t-1}^{(p)}=y_{t-1}$ if $I_{t-1}=1 ; y_{t-1}^{*}$, otherwise, and $y_{2, t-1}^{(p)}=y_{t-1}^{*}$ if $I_{t-1}=1 ; y_{t-1}$, otherwise.

(a) Compute $R_{t}=\left(M_{1 t}+M_{2 t}\right) R_{t-1}$ where $M_{1 t}=\frac{1}{\sigma_{1}} \phi\left(\frac{y_{t}-x_{1 t} \beta_{1}-\lambda_{1}\left(y_{1, t-1}^{(p)}-y_{t-1}\right)}{\sigma_{1}}\right) p_{I_{t-1} 1}$, and $M_{2 t}=\frac{1}{\sigma_{2}} \phi\left(\frac{y_{t}-x_{2 t} \beta_{2}-\lambda_{2}\left(y_{2, t-1}^{(p)}-y_{t-1}\right)}{\sigma_{2}}\right) p_{I_{t-1} 2}$. Simulate $I_{t}$ such that $I_{t}=1$ with probability $M_{1 t} /\left(M_{1 t}+M_{2 t}\right)$ using $u_{t}$.

(b) Simulate $y_{t}^{*}$ as follows:

i. If $I_{t}=1$, simulate $y_{t}^{*}=x_{2 t} \beta_{2}+\lambda_{2}\left(y_{2, t-1}^{(p)}-y_{t-1}\right)+\sigma_{2} \epsilon_{t}$.

ii. If $I_{t}=2$, simulate $y_{t}^{*}=x_{1 t} \beta_{1}+\lambda_{1}\left(y_{1, t-1}^{(p)}-y_{t-1}\right)+\sigma_{1} \epsilon_{t}$.

The above recurrence algorithm can be repeated many times with independently simulated sets of $\epsilon$ and $u$. With $S$ simulation runs, denote $R_{t, j}$ the value of $R_{t}$ for the $j$ th simulation run. The likelihood function can be simulated as $L_{c}=\ln \left(\frac{1}{S} \sum_{j=1}^{S} R_{T, j}\right)$. Other simulated likelihood approaches can similarly be described. ${ }^{2}$

\subsection{Simulated Pseudo Maximum Likelihood Approach}

There are several versions of simulated pseudo maximum likelihood (SPML) approaches introduced in Laroque and Salanie (1993). The one recommended is an approach which uses both simulated first and second order moments in a normal pseudo-likelihood function. Simulate standard normal variables $\epsilon_{1 t}$ and $\epsilon_{2 t}, t=1, \cdots, T$, which are fixed for estimation. Based on (14) and (15), given possible values of parameters and initial values $y_{1,0}^{*}=y_{2,0}^{*}=y_{0}=0, y_{t}$ can be recursively simulated. At $t$, given that $I_{t-1}, y_{1, t-1}^{*}, y_{2, t-1}^{*}$ and $y_{t-1}^{*}$ have been simulated, simulate $I_{t}$ conditional on $I_{t-1}$ with the transition probabilities $p_{12}$ and $p_{21}$. Simulate $y_{1 t}^{*}=x_{1 t} \beta_{1}+\lambda_{1}\left(y_{1, t-1}^{*}-y_{t-1}^{*}\right)+\sigma_{1} \epsilon_{1 t}$ and $y_{2 t}^{*}=x_{2 t} \beta_{2}+\lambda_{2}\left(y_{2, t-1}^{*}-y_{t-1}^{*}\right)+\sigma_{2} \epsilon_{2 t}$. Set $y_{t}^{*}=y_{1 t}^{*}$ if $I_{t}=1$; otherwise, set $y_{t}^{*}=y_{2 t}^{*}$. Denote the simulated value $y_{t}^{*}$ from the sth run by $y_{t}^{*(s)}$. With a total of $S$ runs, the conditional mean and variance of $y_{t}$ can then be simulated by the empirical moments: $E_{t}(\theta)=\frac{1}{S} \sum_{s=1}^{S} y_{t}^{*(s)}$ and $V_{t}(\theta)=$

\footnotetext{
${ }^{2}$ Interested readers can consult Lee (1995) for details.
} 
$\frac{1}{S} \sum_{s=1}^{S}\left(y_{t}^{*(s)}-E_{t}(\theta)\right)^{2}$, where $\theta$ denotes the parameter vector of the model. The SPML estimator is the minimizer of $\frac{1}{2 T} \sum_{t=1}^{T}\left[\left(y_{t}-E_{t}(\theta)\right)^{2} / V_{t}(\theta)+\ln V_{t}(\theta)\right]$. It is possible to generalize this approach by using more moment equations. Let $V_{G t}(\theta)=\frac{1}{S} \sum_{s=1}^{S}\left(y_{t}^{*(s)}-E_{t}(\theta), y_{t-1}^{*(s)}-E_{t-1}(\theta)\right)^{\prime}\left(y_{t}^{*(s)}-\right.$ $\left.E_{t}(\theta), y_{t-1}^{*(s)}-E_{t-1}(\theta)\right)$. A generalized pseudo-likelihood approach (GSPML) can use the pseudo-likelihood $\frac{1}{2(T-1)} \sum_{t=2}^{T}\left[\left(y_{t}-E_{t}(\theta), y_{t-1}-E_{t-1}(\theta)\right) V_{G t}^{-1}(\theta)\left(y_{t}-E_{t}(\theta), y_{t-1}-E_{t-1}(\theta)\right)+\ln \operatorname{det} V_{G t}(\theta)\right] .{ }^{3}$

\subsection{Results of Monte Carlo experiments}

The Markov switching model with (14) and (15) has been estimated by the various simulation methods. We have experimented with different sample sizes $T$ (length of time series) and different numbers of simulation runs $S$. For each case, the number of replications is 400 . The optimization subroutine used for estimation is based on the Powell method (see Press et al. 1986, p.299). A static version (setting $\lambda_{1}=\lambda_{2}=0$ ) is first estimated and its estimates are used as initial starting values for the Powell subroutine. ${ }^{4}$ We report the empirical mean (Mean) and standard deviation (SD) of our estimates for the eight unknown parameters in the model. We report also the number of convergent replications for each case (\# conv) and the average maximized logarithmic simulated likelihood values $(\ln (\mathrm{lk}))$. All the computations are performed in either SUN SPARC 10 or 20 models 50 and 60, or IBM RS/6000 model 580 workstations. The program language is Fortran 77.

Table 1 reports results for the SLSP approach. We have experimented with $T$ from 60 to 200 and $S$ from 50 to 1000 or 2000 . For the case with $T=60$, about 15 replications among the total 400 replications fail to converge. For $T=100$, the number of failure is only 1 to 4 cases. With $T=200$, all replications converge. For all these cases, the estimates of $\beta_{1}$ and $\beta_{2}$ are nearly unbiased. Some biases are in the estimates of $\sigma_{1}$ and $\sigma_{2}$ for $T=60$. These biases decrease as $T$ increases. These estimates are nearly unbiased for sample of size 200. There are obvious downward biases for the estimates of $\lambda_{1}$ and $\lambda_{2}$ and upward biases for the estimates of $p_{12}$ and $p_{21}$. As sample size increases, these biases tend to reduce. Such biases still exist for $T=200$, but are not large. The SD of estimate reduces as sample size increases. The rate of reduction seems to be compatible with the usual $\sqrt{T}$-rate. The estimates are, in general, not sensitive to the number of simulation runs $S$. The biases of the estimates of $\lambda$ 's and $p$ 's tend to reduce with larger $S$ but the chances are small. In terms of CPU time cost for one replication, it takes on average

\footnotetext{
${ }^{3}$ This way of using cross moments of lagged variables has been recommended to me by Professor G. Laroque in personal communication.

${ }^{4}$ Similar results are obtained from different starting values.
} 
29.55 seconds for the case with $T=60$ and $S=100$ in an IBM RS/6000 Model 580 workstation. The CPU time increases to 50.16 seconds for $T=100$ and $S=100 ; 93.21$ seconds for $T=200$ and $S=100$; and 973.15 seconds for $T=200$ and $S=1000$. The CPU time is approximately linear in $T$ or $S .5$

Table 2 reports results on regime classification based on the SLSP simulation method and its estimates in Table 1. Each sample observation is classified to be generated either from regime 1 or from regime 2. Since data are artificially generated, underlying regimes are available. The percentage of overall correct classification as well as separate percentages for each regime are reported. The summary statistics and frequency distributions of correct classification are slightly sensitive to $S$. Classification accuracy improves slightly as $S$ increases. On average (Mean), $72 \%$ of regimes are correctly classified. The statistics of correct overall classification have smaller deviations (SD) than the ones of the separate regime classifications. In terms of frequency distributions for $T=60,50 \%$ of the replications have regimes correctly classified by more than $74 \%$ of periods; $90 \%$ of the replications have regimes correctly classified by more than $60 \%$ of periods. The latter percentage tends to be larger as $T$ increases. With $T=200,90 \%$ of the replications have regimes correctly classified by more than $67 \%$ of periods. ${ }^{6}$ As $T$ increases, the distributions of correct classifications have similar means but variances are reduced.

Finally, results from SPML approaches are reported in Tables 3.1 and 3.2. Except for the estimates of the $\beta$ 's, the performance of the SPML estimates is poor. The estimates of $\lambda$ s are severely biased downward and the estimates of $p$ s are biased upward. The GSPML estimates improve upon the SPML estimates but their biases are still large. Large simulation runs to a thousand do not help much. They. have also serious numerical problems. More than one third of the 400 replications do not converge or converge to boundaries of parameters with zero variances. ${ }^{7}$ The time costs of both the SPML estimates are also larger than the time costs of corresponding SLSP estimates with the same values of $T$ and $S .{ }^{8}$

\footnotetext{
${ }^{5}$ Results from other simulated likelihood approaches which simulate latent states randomly or based on the Markov transition probabilities are rather poor. Biases were much greater under the random selection approach. The biases observed for the randomly selected regime approach were mitigated somewhat when Markov chain probabilities were used to simulate regime paths. On the other hand, the special approach which simulates only the continuous latent variables introduced in Lee (1994) provide slightly better results. These results are reported in Lee (1995).

${ }^{6}$ As the underlying regime probabilities $p_{1}$. and $p_{2}$. are both 0.5 , random guesses would have a $50 \%$ correct classification rate.

${ }^{7}$ Estimates with zero variances are excluded in the reported results.

${ }^{8}$ The simulation of latent states in a SPML approach is based on the Markov transition probabilities (and exogenous variables). Even though the approach of simulating latent states conditional on current and past sample observations provides superior simulated likelihood estimates, such a simulation procedure can not be used for a SPML approach. A pseudo-likelihood approach is, in essential, searching for a nonlinear regression function for the observed dependent variable conditional on exogenous variables. A valid nonlinear regression function will not contain the dependent variable as an regressor. There are also technical difficulties to include observed lagged dependent variables as regressors in a SPML approach for the estimation of latent dynamic models (Laroque and Salanie 1993, p.S123).
} 
Table 1.1

SMLE with Simulated Regime Paths Based on Conditional Prob.(SLSP)

\begin{tabular}{|c|c|c|c|c|c|c|c|c|c|}
\hline $\mathrm{T}$ & S & & Mean & SD & & Mean & SD & \# conv & $\ln (\mathrm{lk})$ \\
\hline \multirow[t]{4}{*}{$\overline{760}$} & $\overline{50}$ & $\overline{\beta_{1}}$ & -1.0272 & $\bar{~} .2363$ & $\overline{\beta_{2}}$ & 1.0202 & $\bar{~} .2598$ & 384 & -99.02 \\
\hline & & $\lambda_{1}$ & .3447 & .2822 & $\lambda_{2}$ & .3458 & .2628 & & \\
\hline & & $\sigma_{1}$ & .9342 & .2485 & $\sigma_{2}$ & .9552 & .2829 & & \\
\hline & & $p_{12}$ & .4036 & .2049 & $p_{21}$ & .4128 & .1892 & & \\
\hline \multirow[t]{4}{*}{60} & 100 & $\beta_{1}$ & -1.0231 & .2306 & $\beta_{2}$ & 1.0211 & .2757 & 384 & -99.11 \\
\hline & & $\lambda_{1}$ & .3550 & .2721 & $\lambda_{2}$ & .3380 & .2669 & & \\
\hline & & $\sigma_{1}$ & .9277 & .2294 & $\sigma_{2}$ & .9595 & .2891 & & \\
\hline & & $p_{12}$ & .3919 & . 1944 & $p_{21}$ & .4044 & .1806 & & \\
\hline \multirow[t]{4}{*}{60} & 500 & $\beta_{1}$ & -1.0185 & .2339 & $\beta_{2}$ & 1.0235 & .2594 & 385 & -99.07 \\
\hline & & $\lambda_{1}$ & .3674 & .2828 & $\lambda_{2}$ & .3406 & .2812 & & \\
\hline & & $\sigma_{1}$ & .9186 & .2332 & $\sigma_{2}$ & .9431 & .2707 & & \\
\hline & & $p_{12}$ & .3978 & 2011 & $p_{21}$ & .4077 & . 1867 & & \\
\hline \multirow[t]{4}{*}{60} & 1000 & $\beta_{1}$ & -1.0236 & .2413 & $\beta_{2}$ & 1.0172 & .2752 & 386 & -99.23 \\
\hline & & $\lambda_{1}$ & .3611 & .2749 & $\lambda_{2}$ & .3452 & .2773 & & \\
\hline & & $\sigma_{1}$ & .9159 & .2313 & $\sigma_{2}$ & .9449 & .2814 & & \\
\hline & & $p_{12}$ & .3932 & .2011 & $p_{21}$ & .4030 & . 1889 & & \\
\hline \multirow[t]{4}{*}{100} & 50 & $\beta_{1}$ & -1.0257 & .1778 & $\beta_{2}$ & 1.0215 & .1844 & 396 & -169.14 \\
\hline & & $\lambda_{1}$ & .4041 & .2079 & $\lambda_{2}$ & .3895 & 1982 & & \\
\hline & & $\sigma_{1}$ & .9843 & .1961 & $\sigma_{2}$ & .9948 & .2142 & & \\
\hline & & $p_{12}$ & .3788 & .1583 & $p_{21}$ & .3777 & .1384 & & \\
\hline \multirow[t]{4}{*}{100} & 100 & $\beta_{1}$ & -1.0241 & .1818 & $\beta_{2}$ & 1.0221 & .1835 & 397 & -168.91 \\
\hline & & $\lambda_{1}$ & .4137 & .2000 & $\lambda_{2}$ & .4056 & .1913 & & \\
\hline & & $\sigma_{1}$ & .9857 & . 1904 & $\sigma_{2}$ & .9791 & .2064 & & \\
\hline & & $p_{12}$ & .3751 & .1540 & $p_{21}$ & .3863 & . 1452 & & \\
\hline \multirow[t]{4}{*}{100} & 500 & $\beta_{1}$ & -1.0258 & .1782 & $\overline{\beta_{2}}$ & 1.0226 & .1785 & 399 & -168.80 \\
\hline & & $\lambda_{1}$ & .4285 & .2068 & $\lambda_{2}$ & .4207 & . 1869 & & \\
\hline & & $\sigma_{1}$ & .9753 & 1932 & $\sigma_{2}$ & .9687 & 2013 & & \\
\hline & & $p_{12}$ & .3656 & .1552 & $p_{21}$ & .3709 & .1417 & & \\
\hline \multirow[t]{4}{*}{100} & 1000 & $\beta_{1}$ & -1.0223 & .1709 & $\beta_{2}$ & 1.0217 & .1786 & 396 & -168.68 \\
\hline & & $\lambda_{1}$ & .4301 & . 1958 & $\lambda_{2}$ & .4418 & . 1830 & & \\
\hline & & $\sigma_{1}$ & .9726 & . 1869 & $\sigma_{2}$ & .9597 & . 1966 & & \\
\hline & & $p_{12}$ & .3577 & .1503 & $p_{21}$ & .3715 & .1460 & & \\
\hline
\end{tabular}

Notes: CPU time per one replication

SLSP: $\mathrm{T}=60, \mathrm{~S}=100,29.55$ seconds; $\mathrm{T}=100, \mathrm{~S}=100,50.16$ seconds. 
Table 1.2

SMLE with Simulated Regime Paths Based on Conditional Prob.(SLSP)

\begin{tabular}{|rr|c|rc|r|rr|r|r|}
\hline $\mathrm{T}$ & $\mathrm{S}$ & & Mean & $\mathrm{SD}$ & & Mean & SD & \# conv & $\ln (\mathrm{lk})$ \\
\hline \hline 200 & 50 & $\beta_{1}$ & -1.0179 & .1236 & $\beta_{2}$ & .9997 & .1225 & 400 & -343.59 \\
& & $\lambda_{1}$ & .4242 & .1199 & $\lambda_{2}$ & .4155 & .1154 & & \\
& & $\sigma_{1}$ & 1.0213 & .1350 & $\sigma_{2}$ & 1.0395 & .1422 & & \\
& & $p_{12}$ & .3796 & .1033 & $p_{21}$ & .3687 & .0952 & & \\
\hline 200 & 100 & $\beta_{1}$ & -1.0207 & .1198 & $\beta_{2}$ & 1.0059 & .1252 & 400 & -343.34 \\
& & $\lambda_{1}$ & .4210 & .1202 & $\lambda_{2}$ & .4229 & .1134 & & \\
& & $\sigma_{1}$ & 1.0200 & .1322 & $\sigma_{2}$ & 1.0363 & .1481 & & \\
& & $p_{12}$ & .3752 & .0989 & $p_{21}$ & .3648 & .0955 & & \\
\hline 200 & 500 & $\beta_{1}$ & -1.0189 & .1179 & $\beta_{2}$ & 1.0024 & .1204 & 400 & -342.57 \\
& & $\lambda_{1}$ & .4399 & .1120 & $\lambda_{2}$ & .4374 & .1020 & & \\
& & $\sigma_{1}$ & 1.0085 & .1295 & $\sigma_{2}$ & 1.0204 & .1331 & & \\
& & $p_{12}$ & .3703 & .1006 & $p_{21}$ & .3580 & .0887 & & \\
\hline 200 & 1000 & $\beta_{1}$ & -1.0193 & .1176 & $\beta_{2}$ & 1.0046 & .1224 & 400 & -342.40 \\
& & $\lambda_{1}$ & .4414 & .1194 & $\lambda_{2}$ & .4382 & .1084 & & \\
& $\sigma_{1}$ & 1.0005 & .1266 & $\sigma_{2}$ & 1.0214 & .1351 & & \\
& $p_{12}$ & .3637 & .1021 & $p_{21}$ & .3555 & .0899 & & \\
\hline 200 & 2000 & $\beta_{1}$ & -1.0186 & .1182 & $\beta_{2}$ & 1.0042 & .1160 & 400 & -342.21 \\
& & $\lambda_{1}$ & .4498 & .1138 & $\lambda_{2}$ & .4465 & .1106 & & \\
& & $\sigma_{1}$ & 1.0010 & .1242 & $\sigma_{2}$ & 1.0103 & .1332 & & \\
& $p_{12}$ & .3644 & .1004 & $p_{21}$ & .3498 & .0873 & & \\
\hline
\end{tabular}

Notes: CPU time per one replication

SLSP: $T=200, S=100,93.21$ seconds; $T=200, S=1000,973.15$ seconds. 
Table 2

Regime Classification - based on Simulated Paths with Conditional Prob.

\begin{tabular}{|c|c|c|c|c|c|c|c|c|}
\hline $\mathrm{T}$ & $\mathrm{S}$ & Mean & $\mathrm{SD}$ & 10\%tile & $25 \%$ tile & median & $75 \%$ tile & $90 \%$ tile \\
\hline \multicolumn{9}{|c|}{ First regime } \\
\hline 60 & 50 & .7133 & .1608 & .4800 & .6036 & .7419 & .8333 & .9032 \\
\hline 60 & 100 & .7222 & .1629 & .5000 & .6071 & .7433 & .8430 & .9211 \\
\hline 60 & 500 & .7300 & .1654 & .5000 & .6216 & .7576 & .8571 & .9333 \\
\hline 60 & 1000 & .7327 & .1672 & .5161 & .6389 & .7581 & .8571 & .9355 \\
\hline 100 & 50 & .7198 & .1315 & .5625 & .6334 & .7363 & .8142 & .8772 \\
\hline 100 & 100 & .7284 & .1282 & .5581 & .6481 & .7442 & .8197 & .8824 \\
\hline 100 & 500 & .7322 & .1357 & .5581 & .6596 & .7500 & .8246 & .8841 \\
\hline 100 & 1000 & .7444 & .1315 & .5714 & .6699 & .7558 & .8443 & .9000 \\
\hline 200 & 50 & .7095 & .0848 & .6009 & .6531 & .7097 & .7688 & .8086 \\
\hline 200 & 100 & .7119 & .0872 & .5974 & .6547 & .7245 & .7684 & .8166 \\
\hline 200 & 500 & .7218 & .0871 & .6211 & .6667 & .7297 & .7756 & .8295 \\
\hline 200 & 1000 & .7257 & .0835 & .6231 & .6733 & .7304 & .7789 & .8319 \\
\hline \multicolumn{9}{|c|}{ Second regime } \\
\hline 60 & 50 & .7108 & .1618 & .5000 & .6000 & .7361 & .8450 & .8974 \\
\hline 60 & 100 & .7200 & .1661 & .5000 & .6207 & .7471 & .8529 & .9048 \\
\hline 60 & 500 & .7206 & .1689 & .4839 & .6154 & .7500 & .8571 & .9130 \\
\hline 60 & 1000 & .7246 & .1724 & .4615 & .6207 & .7586 & .8649 & .9143 \\
\hline 100 & 50 & .7418 & .1309 & .5357 & .6458 & .7375 & .8056 & .8679 \\
\hline 100 & 100 & .7148 & .1391 & .5366 & .6486 & .7381 & .8125 & .8667 \\
\hline 100 & 500 & .7348 & .1431 & .5500 & .6538 & .7555 & .8382 & .9000 \\
\hline 100 & 1000 & .7307 & .1424 & .5319 & .6667 & .7556 & .8275 & .8889 \\
\hline 200 & 50 & .7284 & .0819 & .6274 & .6811 & .7314 & .7838 & .8235 \\
\hline 200 & 100 & .7335 & .0855 & .6220 & .6848 & .7420 & .7915 & .8359 \\
\hline 200 & 500 & .7416 & .0814 & .6296 & .6945 & .7489 & .8000 & .8404 \\
\hline 200 & 1000 & .7406 & .0821 & .6405 & .6923 & .7500 & .7974 & .8359 \\
\hline \multicolumn{9}{|c|}{ overall classification } \\
\hline 60 & 50 & .7202 & .0853 & .6000 & .6667 & .7167 & .7833 & .8333 \\
\hline 60 & 100 & .7307 & .0847 & .6167 & .6833 & .7333 & .7833 & .8333 \\
\hline 60 & 500 & .7350 & .0847 & .6333 & .6667 & .7500 & .8000 & .8333 \\
\hline 60 & 1000 & .7379 & .0844 & .6333 & .6833 & .7500 & .8000 & .8333 \\
\hline 100 & 50 & .7238 & .0617 & .6500 & .6800 & .7250 & .7600 & .8000 \\
\hline 100 & 100 & .7292 & .0609 & .6500 & .6900 & .7300 & .7700 & .8100 \\
\hline 100 & 500 & .7410 & .0585 & .6700 & .7000 & .7400 & .7800 & .8100 \\
\hline 100 & 1000 & .7451 & .0615 & .6700 & .7100 & .7400 & .7900 & .8200 \\
\hline 200 & 50 & .7211 & .0417 & .6700 & .6900 & .7200 & .7500 & .7750 \\
\hline 200 & 100 & .7254 & .0426 & .6750 & .6950 & .7250 & .7550 & .7800 \\
\hline 200 & 500 & .7342 & .0439 & .6800 & .7050 & .7300 & .7650 & .7900 \\
\hline 200 & 1000 & .7357 & .0425 & .6800 & .7050 & .7350 & .7650 & .7850 \\
\hline
\end{tabular}


Table 3.1

Simulated Pseudo MLE

\begin{tabular}{|c|c|c|c|c|c|c|c|c|}
\hline $\mathrm{T}$ & $\mathrm{S}$ & & Mean & SD & & Mean & SD & \# conv \\
\hline \multicolumn{9}{|c|}{ Simulated Pseudo MLE (SPML) } \\
\hline \multirow[t]{4}{*}{60} & 100 & $\beta_{1}$ & -1.0289 & .3141 & $\beta_{2}$ & 1.0289 & .3340 & 231 \\
\hline & & $\lambda_{1}$ & .1784 & .3737 & $\lambda_{2}$ & .0994 & .3201 & \\
\hline & & $\sigma_{1}$ & .9161 & .5018 & $\sigma_{2}$ & .8890 & .5145 & \\
\hline & & $p_{12}$ & .5586 & .2300 & $p_{21}$ & .5470 & .2080 & \\
\hline \multirow[t]{4}{*}{60} & 200 & $\overline{\beta_{1}}$ & -1.0335 & .3289 & $\beta_{2}$ & .9997 & .3498 & 222 \\
\hline & & $\lambda_{1}$ & .1875 & .3436 & $\lambda_{2}$ & 1294 & .3374 & \\
\hline & & $\sigma_{1}$ & .9117 & .4902 & $\sigma_{2}$ & .8826 & .5930 & \\
\hline & & $p_{12}$ & .6001 & .2266 & $p_{21}$ & .5729 & .1953 & \\
\hline & 500 & $\beta_{1}$ & -1.0262 & .3142 & $\beta_{2}$ & 1.0484 & .3540 & 239 \\
\hline & & $\lambda_{1}$ & .1519 & .3543 & $\lambda_{2}$ & .1439 & .3259 & \\
\hline & & $\sigma_{1}$ & .8405 & 4914 & $\sigma_{2}$ & .9031 & .4764 & \\
\hline & & $p_{12}$ & .5603 & .2336 & $p_{21}$ & .5819 & 2089 & \\
\hline & 1000 & $\beta_{1}$ & -1.0447 & .3241 & $\beta_{2}$ & 1.0418 & .3402 & 253 \\
\hline & & $\lambda_{1}$ & .1814 & .3341 & $\lambda_{2}$ & .1707 & .3357 & \\
\hline & & $\sigma_{1}$ & .8667 & .4665 & $\sigma_{2}$ & .8606 & .5228 & \\
\hline & & $p_{12}$ & .5628 & .2268 & $p_{21}$ & .5710 & .2043 & \\
\hline \multirow[t]{4}{*}{100} & 100 & $\overline{\beta_{1}}$ & -1.0226 & .2457 & $\overline{\overline{\beta_{2}}}$ & 1.0282 & .2647 & 254 \\
\hline & & $\lambda_{1}$ & .1793 & .3629 & $\lambda_{2}$ & .2142 & .2754 & \\
\hline & & $\sigma_{1}$ & .9451 & .4734 & $\sigma_{2}$ & .9170 & .4365 & \\
\hline & & $p_{12}$ & .5662 & .2065 & $p_{21}$ & .5522 & .1956 & \\
\hline \multirow[t]{4}{*}{100} & 200 & $\beta_{1}$ & -1.0199 & .2361 & $\beta_{2}$ & 1.0393 & .2472 & 247 \\
\hline & & $\lambda_{1}$ & . 1732 & .3652 & $\lambda_{2}$ & .1961 & .3025 & \\
\hline & & $\sigma_{1}$ & .9125 & .4267 & $\sigma_{2}$ & .9471 & .4967 & \\
\hline & & $p_{12}$ & .5406 & .2030 & $p_{21}$ & .5569 & .1801 & \\
\hline \multirow{4}{*}{\multicolumn{2}{|c|}{100}} & $\beta_{1}$ & -1.0257 & .2396 & $\beta_{2}$ & 1.0292 & .2549 & 265 \\
\hline & & $\lambda_{1}$ & .2149 & .3144 & $\lambda_{2}$ & .2162 & .2765 & \\
\hline & & $\sigma_{1}$ & .8885 & .4653 & $\sigma_{2}$ & .9527 & .4558 & \\
\hline & & $p_{12}$ & .5607 & .2051 & $p_{21}$ & .5632 & .1825 & \\
\hline \multirow[t]{4}{*}{100} & 1000 & $\beta_{1}$ & -1.0158 & .2400 & $\beta_{2}$ & 1.0398 & .2494 & 282 \\
\hline & & $\lambda_{1}$ & .2070 & .3461 & $\lambda_{2}$ & .2106 & .2972 & \\
\hline & & $\sigma_{1}$ & .9079 & .4923 & $\sigma_{2}$ & .8924 & .4575 & \\
\hline & & $p_{12}$ & .5280 & .1903 & $p_{21}$ & .5556 & .1691 & \\
\hline
\end{tabular}

Notes: CPU time per one replication

SPML: $\mathrm{T}=100, \mathrm{~S}=500,421.63$ seconds; $\mathrm{T}=60, \mathrm{~S}=1000,539.92$ seconds. 
Table 3.2

Simulated Pseudo MLE

\begin{tabular}{|c|c|c|c|c|c|c|c|c|}
\hline $\mathrm{T}$ & S & & Mean & SD & & Mean & SD & \# conv \\
\hline \multicolumn{9}{|c|}{ Generalized Simulated Pseudo MLE (GSPML) } \\
\hline \multirow[t]{4}{*}{60} & 100 & $\beta_{1}$ & -1.0011 & .3005 & $\bar{\beta}_{2}$ & 1.0106 & .3324 & 232 \\
\hline & & $\lambda_{1}$ & .2961 & .3360 & $\lambda_{2}$ & .2515 & .3156 & \\
\hline & & $\sigma_{1}$ & .8943 & .5112 & $\sigma_{2}$ & .8626 & .4722 & \\
\hline & & $p_{12}$ & . 4930 & .2134 & $p_{21}$ & .4971 & .2027 & \\
\hline \multirow[t]{4}{*}{60} & 200 & $\beta_{1}$ & -1.0415 & .3308 & $\beta_{2}$ & 1.0131 & .2916 & 238 \\
\hline & & $\lambda_{1}$ & .2786 & .3209 & $\lambda_{2}$ & .2581 & .2866 & \\
\hline & & $\sigma_{1}$ & .9058 & .4372 & $\sigma_{2}$ & .8682 & .4771 & \\
\hline & & $p_{12}$ & .5049 & .2165 & $p_{21}$ & .4886 & .1720 & \\
\hline \multirow[t]{4}{*}{60} & 500 & $\beta_{1}$ & -1.0267 & .2910 & $\beta_{2}$ & 1.0459 & .3162 & 243 \\
\hline & & $\lambda_{1}$ & .2944 & 3130 & $\lambda_{2}$ & .2907 & .2874 & \\
\hline & & $\sigma_{1}$ & .8548 & .4689 & $\sigma_{2}$ & .8547 & .4907 & \\
\hline & & $p_{12}$ & .4730 & .1939 & $p_{21}$ & .5103 & .2000 & \\
\hline \multirow[t]{4}{*}{60} & 1000 & $\beta_{1}$ & -1.0203 & 3001 & $\beta_{2}$ & 1.0378 & .3197 & 238 \\
\hline & & $\lambda_{1}$ & .2986 & 2831 & $\lambda_{2}$ & .2932 & .2755 & \\
\hline & & $\sigma_{1}$ & .8619 & .4842 & $\sigma_{2}$ & .8733 & .5255 & \\
\hline & & $p_{12}$ & .4761 & 2008 & $p_{21}$ & .5040 & .1908 & \\
\hline \multirow[t]{4}{*}{100} & 100 & $\beta_{1}$ & -1.0428 & .2446 & $\overline{\beta_{2}}$ & 1.0093 & .2545 & 238 \\
\hline & & $\lambda_{1}$ & .3580 & .2592 & $\lambda_{2}$ & .3188 & .2349 & \\
\hline & & $\sigma_{1}$ & .9822 & .4265 & $\sigma_{2}$ & .8584 & . .4216 & \\
\hline & & $p_{12}$ & .5032 & 1968 & $p_{21}$ & . 4717 & .1630 & \\
\hline \multirow[t]{4}{*}{100} & 200 & $\beta_{1}$ & -1.0128 & 2178 & $\beta_{2}$ & 1.0315 & .2296 & 251 \\
\hline & & $\lambda_{1}$ & .3260 & 2790 & $\lambda_{2}$ & .3387 & .2452 & \\
\hline & & $\sigma_{1}$ & .8885 & .4296 & $\sigma_{2}$ & .9143 & .4892 & \\
\hline & & $p_{12}$ & .4576 & .1752 & $p_{21}$ & .4936 & .1743 & \\
\hline \multirow[t]{4}{*}{100} & 500 & $\beta_{1}$ & -1.0255 & .2183 & $\bar{\beta} \beta_{2}$ & 1.0256 & 2374 & 271 \\
\hline & & $\lambda_{1}$ & .3677 & .2527 & $\lambda_{2}$ & .3442 & 2311 & \\
\hline & & $\sigma_{1}$ & .8926 & .4264 & $\sigma_{2}$ & .8654 & 4395 & \\
\hline & & $p_{12}$ & .4565 & . 1700 & $p_{21}$ & .4712 & .1675 & \\
\hline \multirow[t]{4}{*}{100} & 1000 & $\beta_{1}$ & -1.0058 & . 2158 & $\beta_{2}$ & 1.0418 & .2380 & 275 \\
\hline & & $\lambda_{1}$ & .3562 & .2565 & $\lambda_{2}$ & .3536 & .2347 & \\
\hline & & $\sigma_{1}$ & 8673 & .4379 & $\sigma_{2}$ & .9298 & 4158 & \\
\hline & & $p_{12}$ & .4439 & .1753 & $p_{21}$ & .4585 & .1471 & \\
\hline
\end{tabular}

Notes: CPU time per one replication

GSPML: $\mathrm{T}=60, \mathrm{~S}=200,157.84$ seconds; $\mathrm{T}=100, \mathrm{~S}=200,263.81$ seconds; $\mathrm{T}=100, \mathrm{~S}=1000,1387.50$ seconds. 


\section{Monte Carlo Studies On Dynamic Disequilibrium Model}

Sample data for the dynamic disequilibrium model are generated from equations:

$$
\begin{aligned}
& y_{1 t}^{*}=x_{1 t} \beta_{1}+\lambda_{1}\left(y_{1, t-1}^{*}-y_{t-1}\right)+\sigma_{1} \epsilon_{1 t}, \\
& y_{2 t}^{*}=x_{2 t} \beta_{2}+\lambda_{2}\left(y_{2, t-1}^{*}-y_{t-1}\right)+\sigma_{2} \epsilon_{2 t},
\end{aligned}
$$

and

$$
y_{t}=\min \left(y_{1 t}^{*}, y_{2 t}^{*}\right)
$$

where $\epsilon_{1 t}$ and $\epsilon_{2 t}$ are independent of each other and are i.i.d. $N(0,1)$ for all $t$. This model has six parameters. The true parameters are set to $\beta_{1}=-1, \lambda_{1}=0.5, \sigma_{1}=1, \beta_{2}=1, \lambda_{2}=0.5$, and $\sigma_{2}=1$.

\subsection{Simulation with Simulated Regime Paths based on Conditional Sample Probabilities}

The simulated likelihood of the SLSP approach is $h_{c}$ in (11). The detailed simulation steps for this special model are as follows.

Simulate two sets of uniform random variables $u_{t}$ and $v_{t}, t=1, \ldots, T$, on $[0,1]$, which are fixed and invariant of unknown parameters during estimation.

1. For period 1:

(a) Compute $R_{1}=M_{11}+M_{21}$ where $M_{11}=\frac{1}{\sigma_{1}} \phi\left(\frac{y_{1}-x_{11} \beta_{1}}{\sigma_{1}}\right) \cdot \Phi\left(-\frac{y_{1}-x_{21} \beta_{2}}{\sigma_{2}}\right)$, and $M_{21}=$ $\frac{1}{\sigma_{2}} \phi\left(\frac{y_{1}-x_{21} \beta_{2}}{\sigma_{2}}\right) \cdot \Phi\left(-\frac{y_{1}-x_{11} \beta_{1}}{\sigma_{1}}\right)$.

(b) Simulate regime indicator $I_{1}$ such that $I_{1}=1$ with the probability $M_{11} /\left(M_{11}+M_{21}\right)$ using $v_{1}$.

(c) Simulate $y_{1}^{*}=x_{21} \beta_{2}-\sigma_{2} \Phi^{-1}\left[u_{1} \Phi\left(-\frac{y_{1}-x_{21} \beta_{2}}{\sigma_{2}}\right)\right]$ if $I_{1}=1$, but simulate $y_{1}^{*}=x_{11} \beta_{1}-$ $\sigma_{1} \Phi^{-1}\left[u_{1} \Phi\left(-\frac{y_{1}-x_{11} \beta_{1}}{\sigma_{1}}\right)\right]$ if $I_{1}=2$.

2. For period $t$ :

The $I_{t-1}$ and $y_{t-1}^{*}$ have been simulated in the previous steps. $R_{t-1}$ has also been evaluated. Define $y_{1, t-1}^{(p)}$ and $y_{2, t-1}^{(p)}$ such that $y_{1, t-1}^{(p)}=y_{t-1}$ if $I_{t-1}=1 ; y_{t-1}^{*}$, otherwise, and $y_{2, t-1}^{(p)}=y_{t-1}^{*}$ if $I_{t-1}=1$; $y_{t-1}$, otherwise.

(a) Compute $R_{t}=\left(M_{1 t}+M_{2 t}\right) R_{t-1}$ where

$$
M_{1 t}=\frac{1}{\sigma_{1}} \phi\left(\frac{y_{t}-x_{1 t} \beta_{1}-\lambda_{1}\left(y_{1, t-1}^{(p)}-y_{t-1}\right)}{\sigma_{1}}\right) \cdot \Phi\left(-\frac{y_{t}-x_{2 t} \beta_{2}-\lambda_{2}\left(y_{2, t-1}^{(p)}-y_{t-1}\right)}{\sigma_{2}}\right),
$$


and $M_{2 t}=\frac{1}{\sigma_{2}} \phi\left(\frac{y_{t}-x_{2 t} \beta_{2}-\lambda_{2}\left(y_{2, t-1}^{(p)}-y_{t-1}\right)}{\sigma_{2}}\right) \cdot \Phi\left(-\frac{y_{t}-x_{1 t} \beta_{1}-\lambda_{1}\left(y_{1, t-1}^{(p)}-y_{t-1}\right)}{\sigma_{1}}\right)$. Simulate $I_{t}$ such that $I_{t}=1$ with probability $M_{1 t} /\left(M_{1 t}+M_{2 t}\right)$ using $v_{t}$.

(b) Simulate $y_{t}^{*}$ as follows:

i. If $I_{t}=1$, simulate a truncated normal variable $\epsilon_{t}=-\left[u_{t} \Phi\left(-\frac{y_{t}-x_{2 t} \beta_{2}-\lambda_{2}\left(y_{2, t-1}^{(p)}-y_{t-1}\right)}{\sigma_{2}}\right)\right]$ and simulate $y_{t}^{*}=x_{2 t} \beta_{2}+\lambda_{2}\left(y_{2, t-1}^{(p)}-y_{t-1}\right)+\sigma_{2} \epsilon_{t}$.

ii. If $I_{t}=2$, simulate $\epsilon_{t}=-\Phi^{-1}\left[u_{t} \Phi\left(-\frac{y_{t}-x_{1 t} \beta_{1}-\lambda_{1}\left(y_{1, t-1}^{(p)}-y_{t-1}\right)}{\sigma_{1}}\right)\right]$ and $y_{t}^{*}=x_{1 t} \beta_{1}+$ $\lambda_{1}\left(y_{1, t-1}^{(p)}-y_{t-1}\right)+\sigma_{1} \epsilon_{t}$.

With $S$ simulation runs, denote $R_{t, j}$ the simulated value of $R_{t}$ for the $j$ th simulation run. The simulated likelihood function is $L_{c}=\ln \left(\frac{1}{S} \sum_{j=1}^{S} R_{T, j}\right)$.

\subsection{Simulated Pseudo Maximum Likelihood Approach}

The SPML approaches will simulate the model as in (16-18). At $t$, given that $y_{i, t-1}^{*}$ for $i=1,2$ and $y_{t-1}^{*}$ have been simulated, simulate $y_{i t}^{*}=x_{i t} \beta_{i}+\lambda_{i}\left(y_{i, t-1}^{*}-y_{t-1}^{*}\right)+\sigma_{i} \epsilon_{i t}$ for $i=1$ and 2 . Set $y_{t}^{*}=\min \left\{y_{1 t}^{*}, y_{2 t}^{*}\right\}$. With $S$ simulation runs, let $E_{t}(\theta)=\frac{1}{S} \sum_{s=1}^{S} y_{t}^{*(s)}, V_{t}(\theta)=\frac{1}{S} \sum_{s=1}^{S}\left(y_{t}^{*(s)}-E_{t}(\theta)\right)^{2}$, and $V_{G t}(\theta)=\frac{1}{S} \sum_{s=1}^{S}\left(y_{t}^{*(s)}-E_{t}(\theta), y_{t-1}^{*(s)}-E_{t-1}(\theta)\right)^{\prime}\left(y_{t}^{*(s)}-E_{t}(\theta), y_{t-1}^{*(s)}-E_{t-1}(\theta)\right)$. The SPML approach uses the simulated pseudo-likelihood $\frac{1}{2 T} \sum_{t=1}^{T}\left[\left(y_{t}-E_{t}(\theta)\right)^{2} / V_{t}(\theta)+\ln V_{t}(\theta)\right]$. The GSPML approach uses the pseudo-likelihood $\frac{1}{2(T-1)} \sum_{t=2}^{T}\left[\left(y_{t}-E_{t}(\theta), y_{t-1}-E_{t-1}(\theta)\right) V_{G t}^{-1}(\theta)\left(y_{t}-E_{t}(\theta), y_{t-1}-E_{t-1}(\theta)\right)+\right.$ $\left.\ln \operatorname{det} V_{G t}(\theta)\right]$.

\subsection{Monte Carlo Results}

As this model has a smaller number of parameters, sample sizes $T$ for our experiments vary from 30 to 100 . The number of simulation runs $S$ varies from 50 to 200 . For each case, the number of replications is 400 .

Table 4 reports results for the SLSP approach. For $T=30$, the optimization subroutine fails to converge with 8 or 9 per 400 replications. For $T=60$, the number of failure is only 1 when $m=50$. With $T=100$, all replications converge. In all these cases, estimates of $\beta_{1}$ are nearly unbiased. There are some downward biases in the estimates of the remaining parameters. The magnitudes of these biases decrease as $T$ increases. For $T=60$ or 100 , the biases are quite small. The estimates are insensitive to $S$. The insensitivity shows up also in the reported likelihood values $\ln (l k)$. Simulation runs with $S=50$ are, in general, good enough. In terms of CPU time cost, it takes about 98.99 seconds on average to complete one replication for the case with $T=60$ and $S=200$. Table 5 report results on regime classification. 
Classification accuracy is insensitive to $S$. On average (Mean), about $77 \%$ of the regimes are correctly classified. In terms of frequency distributions, $50 \%$ of the replications have correctly classified regimes by more than $77 \%$ of periods. For $T=60$ or $100,90 \%$ of the replications have correctly classified regimes by more than $70 \%$ of periods. The latter percentage is about $63 \%$ for $T=30$.

Tables 6.1 and 6.2 report SPML estimates. The SPML estimates are also not sensitive to $S$. Results with thousand simulation runs are similar to results with a few hundred runs. The biases of the SPML estimates are similar to the ones of the SLSP estimates except those of the estimates of $\sigma_{1}$. The SPML estimates of $\sigma_{1}$ have larger downward biases. The GSPML estimates improve upon the SPML estimates and are comparable with the SLSP estimates in term of biases. The main difference is that both the SPML and GSPML have larger variances than the SLSP estimates. On the other hand, the CPU time cost of the SPML approach for this model is inexpensive. The time cost of the GSPML approach is much more expensive than the SPML approach but is still relatively less than the SLSP approach. This is so because the SLSP approach involves the computation of the normal distribution and its inverse function while both the SPML and GSPML do not. For the disequilibrium model, these pseudo-likelihood approaches are numerically stable except for the case with small sample size. For $T=30$, slightly more than $5 \%$ of replications do not converge. In comparison, the simulated pseudo-likelihood approaches behave much better for estimation of the disequilibrium model than the switching regression model. 
Table 4

Dynamic Disequilibrium Model

SMLE with Simulated Regime Paths Based on Conditional Prob.(SLSP)

\begin{tabular}{|rr|r|rr|r|rr|r|r|}
\hline $\mathrm{T}$ & $\mathrm{S}$ & & Mean & $\mathrm{SD}$ & & Mean & SD & \# conv & $\ln (\mathrm{lk})$ \\
\hline \hline 30 & 50 & $\beta_{1}$ & -.9991 & .2851 & $\beta_{2}$ & .9525 & .2869 & 391 & -37.54 \\
& & $\lambda_{1}$ & .4466 & .2777 & $\lambda_{2}$ & .4236 & .2816 & & \\
& & $\sigma_{1}$ & .8724 & .2815 & $\sigma_{2}$ & .9621 & .2536 & & \\
\hline 30 & 100 & $\beta_{1}$ & -1.0046 & .2918 & $\beta_{2}$ & .9502 & .2843 & 393 & -37.50 \\
& & $\lambda_{1}$ & .4516 & .2826 & $\lambda_{2}$ & .4276 & .2793 & & \\
& & $\sigma_{1}$ & .8581 & .2733 & $\sigma_{2}$ & .9601 & .2469 & & \\
\hline 30 & 200 & $\beta_{1}$ & -1.0028 & .2929 & $\beta_{2}$ & .9654 & .2931 & 392 & -37.53 \\
& & $\lambda_{1}$ & .4494 & .2711 & $\lambda_{2}$ & .4249 & .2762 & & \\
& & $\sigma_{1}$ & .8557 & .2710 & $\sigma_{2}$ & .9655 & .2520 & & \\
\hline \hline 60 & 50 & $\beta_{1}$ & -1.0073 & .1850 & $\beta_{2}$ & .9737 & .1807 & 399 & -78.88 \\
& & $\lambda_{1}$ & .4952 & .1758 & $\lambda_{2}$ & .4727 & .1814 & & \\
& & $\sigma_{1}$ & .9439 & .1679 & $\sigma_{2}$ & .9917 & .1608 & & \\
\hline 60 & 100 & $\beta_{1}$ & -1.0068 & .1836 & $\beta_{2}$ & .9788 & .1777 & 400 & -78.85 \\
& & $\lambda_{1}$ & .4888 & .1754 & $\lambda_{2}$ & .4780 & .1650 & & \\
& & $\sigma_{1}$ & .9434 & .1744 & $\sigma_{2}$ & .9922 & .1688 & & \\
\hline 60 & 200 & $\beta_{1}$ & -1.0078 & .1812 & $\beta_{2}$ & .9746 & .1752 & 400 & -78.85 \\
& & $\lambda_{1}$ & .4922 & .1685 & $\lambda_{2}$ & .4820 & .1638 & & \\
& & $\sigma_{1}$ & .9405 & .1708 & $\sigma_{2}$ & .9890 & .1599 & & \\
\hline \hline 100 & 50 & $\beta_{1}$ & -1.0003 & .1365 & $\beta_{2}$ & .9610 & .1331 & 400 & -133.45 \\
& & $\lambda_{1}$ & .5022 & .1177 & $\lambda_{2}$ & .4781 & .1219 & & \\
& & $\sigma_{1}$ & .9736 & .1376 & $\sigma_{2}$ & 1.0112 & .1387 & & \\
\hline 100 & 100 & $\beta_{1}$ & -1.0037 & .1375 & $\beta_{2}$ & .9577 & .1342 & 400 & -133.29 \\
& & $\lambda_{1}$ & .5066 & .1146 & $\lambda_{2}$ & .4847 & .1272 & & \\
& & $\sigma_{1}$ & .9686 & .1406 & $\sigma_{2}$ & 1.0083 & .1417 & & \\
\hline 100 & 200 & $\beta_{1}$ & -1.0038 & .1309 & $\beta_{2}$ & .9661 & .1330 & 400 & -133.28 \\
& & $\lambda_{1}$ & .5042 & .1117 & $\lambda_{2}$ & .4822 & .1209 & & \\
& $\sigma_{1}$ & .9670 & .1364 & $\sigma_{2}$ & 1.0002 & .1360 & & \\
\hline
\end{tabular}

Notes: CPU time per one replication

SLSP: $\mathrm{T}=60, \mathrm{~S}=200,98.99$ seconds. 
Table 5

Dynamic Disequilibrium Model

Regime Classification - based on Simulated Regimes with Conditional Prob.

\begin{tabular}{|c|c|c|c|c|c|c|c|c|}
\hline $\mathrm{T}$ & $\mathrm{S}$ & Mean & $\mathrm{SD}$ & 10\%tile & $25 \%$ tile & median & $75 \%$ tile & $90 \%$ tile \\
\hline \multicolumn{9}{|c|}{ regime 1} \\
\hline 30 & 50 & .7547 & .1721 & .5333 & .6667 & .7857 & .8824 & .9412 \\
\hline 30 & 100 & .7516 & .1731 & .5333 & .6667 & .7692 & .8824 & .9474 \\
\hline 30 & 200 & .7591 & .1697 & .5455 & .6743 & .7857 & .8750 & .9444 \\
\hline 60 & 50 & .7806 & .1087 & .6452 & .7097 & .7931 & .8611 & .9062 \\
\hline 60 & 100 & .7773 & .1099 & .6376 & .7143 & .7879 & .8571 & .9091 \\
\hline 60 & 200 & .7888 & .1104 & .6405 & .7222 & .8065 & .8750 & .9200 \\
\hline 100 & 50 & .7581 & .0894 & .6373 & .7034 & .7600 & .8207 & .8717 \\
\hline 100 & 100 & .7641 & .0876 & .6523 & .7111 & .7708 & .8240 & .8794 \\
\hline 100 & 200 & .7692 & .0865 & .6667 & .7113 & .7708 & .8300 & .8864 \\
\hline \multicolumn{9}{|c|}{ regime 2} \\
\hline 30 & 50 & .7639 & .1656 & .5385 & .6667 & .8000 & .8889 & .9474 \\
\hline 30 & 100 & .7652 & .1652 & .5455 & .6875 & .7895 & .8889 & .9444 \\
\hline 30 & 200 & .7667 & .1601 & .5385 & .6667 & .8000 & .8824 & .9444 \\
\hline 60 & 50 & .7764 & .1120 & .6429 & .7097 & .7812 & .8571 & .9130 \\
\hline 60 & 100 & .7768 & .1085 & .6452 & .7165 & .7868 & .8524 & .9105 \\
\hline 60 & 200 & .7779 & .1092 & .6364 & .7143 & .7879 & .8621 & .9091 \\
\hline 100 & 50 & .7726 & .0816 & .6643 & .7216 & .7788 & .8304 & .8714 \\
\hline 100 & 100 & .7750 & .0797 & .6667 & .7176 & .7835 & .8333 & .8692 \\
\hline 100 & 200 & .7825 & .0795 & .6809 & .7253 & .7864 & .8438 & .8864 \\
\hline \multicolumn{9}{|c|}{ overall classification } \\
\hline 30 & 50 & .7662 & .0920 & .6333 & .7000 & .7667 & .8333 & .8667 \\
\hline 30 & 100 & .7659 & .0868 & .6333 & .7000 & .7667 & .8333 & .8667 \\
\hline 30 & 200 & .7696 & .0872 & .6667 & .7000 & .7667 & .8333 & .8667 \\
\hline 60 & 50 & .7829 & .0582 & .7000 & .7500 & .7833 & .8333 & .8500 \\
\hline 60 & 100 & .7802 & .0592 & .7000 & .7500 & .7833 & .8167 & .8500 \\
\hline 60 & 200 & .7880 & .0576 & .7167 & .7500 & .7833 & .8333 & .8667 \\
\hline 100 & 50 & .7669 & .0495 & .7000 & .7300 & .7700 & .8000 & .8300 \\
\hline 100 & 100 & .7709 & .0527 & .7000 & .7400 & .7700 & .8000 & .8400 \\
\hline 100 & 200 & .7777 & .0521 & .7100 & .7400 & .7800 & .8150 & .8400 \\
\hline
\end{tabular}


Table 6.1

SPMLE of Dynamic Disequilibrium Model

True parameters: $\beta_{d}=-1, \lambda_{d}=0.5, \sigma_{d}=1$ and $\beta_{s}=1, \lambda_{s}=0.5, \sigma_{s}=1$

\begin{tabular}{|c|c|c|c|c|c|c|c|c|}
\hline $\mathrm{T}$ & & Mean & SD & & Mean & $\mathrm{SD}$ & \multirow{2}{*}{$\frac{\text { SPML }}{-10.69}$} & \#conv \\
\hline \multirow[t]{3}{*}{30} & $\overline{\beta_{1}}$ & -.9836 & .3666 & $\overline{\beta_{2}}$ & .9811 & .3112 & & 372 \\
\hline & $\lambda_{1}$ & .4171 & .3579 & $\lambda_{2}$ & .4180 & .3143 & & \\
\hline & $\sigma_{1}$ & .8830 & .3906 & $\sigma_{2}$ & .9453 & .3407 & & \\
\hline \multirow[t]{3}{*}{30} & $\beta_{1}$ & -.9985 & .4152 & $\beta_{2}$ & .9648 & .3210 & -10.71 & 373 \\
\hline & $\lambda_{1}$ & .4175 & .3333 & $\lambda_{2}$ & .4346 & .2691 & & \\
\hline & $\sigma_{1}$ & .8550 & .3634 & $\sigma_{2}$ & .9637 & .3340 & & \\
\hline 100 & $\overline{\beta_{1}}$ & -1.0046 & .3285 & $\beta_{2}$ & .9856 & .3226 & -10.33 & 368 \\
\hline & $\lambda_{1}$ & .4313 & .3282 & $\lambda_{2}$ & .4357 & .2865 & & \\
\hline & $\sigma_{1}$ & .8394 & .3877 & $\sigma_{2}$ & .9521 & .3292 & & \\
\hline \multirow[t]{3}{*}{30} & $\overline{\beta_{1}}$ & -1.0098 & .3703 & $\beta_{2}$ & .9752 & .3082 & -10.43 & 382 \\
\hline & $\lambda_{1}$ & .4487 & .3072 & $\lambda_{2}$ & .4293 & .2897 & & \\
\hline & $\sigma_{1}$ & .8117 & .3543 & $\sigma_{2}$ & .9372 & .3339 & & \\
\hline \multirow[t]{3}{*}{30} & $\overline{\beta_{1}}$ & -1.0256 & .3646 & $\overline{\beta_{2}}$ & .9805 & .3044 & -10.23 & 379 \\
\hline & $\lambda_{1}$ & .4384 & .3206 & $\lambda_{2}$ & .4357 & .2885 & & \\
\hline & $\sigma_{1}$ & .8011 & .3776 & $\sigma_{2}$ & .9321 & .3337 & & \\
\hline \multirow[t]{3}{*}{30} & $\overline{\beta_{1}}$ & -1.0198 & .3456 & $\beta_{2}$ & .9846 & .3212 & -10.30 & 372 \\
\hline & $\lambda_{1}$ & .4343 & .3169 & $\lambda_{2}$ & .4364 & .2720 & & \\
\hline & $\sigma_{1}$ & .8083 & .3551 & $\sigma_{2}$ & .9315 & .3399 & & \\
\hline \multirow[t]{2}{*}{100} & $\overline{\beta_{1}}$ & -1.0112 & .1436 & $\beta_{2}$ & .9774 & .1507 & -46.15 & 399 \\
\hline & $\lambda_{1}$ & .5032 & .1467 & $\lambda_{2}$ & .4831 & .1751 & & \\
\hline & $\sigma_{1}$ & .9697 & .1631 & $\sigma_{2}$ & 1.0393 & .1701 & & \\
\hline \multirow[t]{3}{*}{100} & $\beta_{1}$ & -1.0077 & .1390 & $\beta_{2}$ & .9891 & .1441 & -45.11 & 400 \\
\hline & $\lambda_{1}$ & .4994 & .1289 & $\lambda_{2}$ & .4864 & .1298 & & \\
\hline & $\sigma_{1}$ & .9589 & .1547 & $\sigma_{2}$ & 1.0291 & .1672 & & \\
\hline \multirow[t]{3}{*}{100} & $\overline{\beta_{1}}$ & -1.0108 & .1374 & $\beta_{2}$ & .9860 & .1408 & -44.06 & 400 \\
\hline & $\lambda_{1}$ & .4972 & .1266 & $\lambda_{2}$ & .4809 & .1428 & & \\
\hline & $\sigma_{1}$ & .9496 & .1495 & $\sigma_{2}$ & 1.0148 & .1709 & & \\
\hline \multirow[t]{3}{*}{$100 \quad 500$} & $\beta_{1}$ & -1.0113 & .1355 & $\beta_{2}$ & .9883 & .1375 & -43.42 & 400 \\
\hline & $\lambda_{1}$ & .4973 & .1224 & $\lambda_{2}$ & .4884 & .1250 & & \\
\hline & $\sigma_{1}$ & .9419 & .1461 & $\sigma_{2}$ & 1.0067 & .1597 & & \\
\hline \multirow[t]{3}{*}{$100 \quad 1000$} & $\overline{\beta_{1}}$ & -1.0098 & .1359 & $\overline{\beta_{2}}$ & .9877 & .1396 & -43.33 & 400 \\
\hline & $\lambda_{1}$ & .4981 & .1238 & $\lambda_{2}$ & .4871 & .1272 & & \\
\hline & $\sigma_{1}$ & .9417 & .1494 & $\sigma_{2}$ & 1.0042 & .1677 & & \\
\hline \multirow[t]{3}{*}{$100 \quad 3000$} & $\beta_{1}$ & -1.0100 & .1354 & $\overline{\beta_{2}}$ & .9887 & .1385 & -43.34 & 400 \\
\hline & $\lambda_{1}$ & .4966 & .1221 & $\lambda_{2}$ & .4874 & .1239 & & \\
\hline & $\sigma_{1}$ & .9412 & .1459 & $\sigma_{2}$ & 1.0076 & .1578 & & \\
\hline
\end{tabular}

Notes: CPU time per one replication

SPML: $\mathrm{T}=30, \mathrm{~S}=100,3.32$ seconds; $\mathrm{T}=30, \mathrm{~S}=500,16.68$ seconds; $\mathrm{T}=100, \mathrm{~S}=100,9.35$ seconds; $\mathrm{T}=100, \mathrm{~S}=500,45.28$ seconds. 
Table 6.2

SPMLE of Dynamic Disequilibrium Model

True parameters: $\beta_{d}=-1, \lambda_{d}=0.5, \sigma_{d}=1$ and $\beta_{s}=1, \lambda_{s}=0.5, \sigma_{s}=1$

\begin{tabular}{|c|c|c|c|c|c|c|c|c|c|}
\hline 1 & $\mathrm{~S}$ & & Mean & SD & & Mean & SD & SPML & \#conv \\
\hline \multicolumn{10}{|c|}{ Generalized Simulated Pseudo MLE (GSPML) } \\
\hline \multirow[t]{3}{*}{60} & 30 & $\beta_{1}$ & -1.0144 & .2077 & $\beta_{2}$ & .9886 & .2075 & -26.41 & 396 \\
\hline & & $\lambda_{1}$ & .4807 & .2167 & $\lambda_{2}$ & .4589 & .2219 & & \\
\hline & & $\sigma_{1}$ & .9246 & 2271 & $\sigma_{2}$ & 1.0328 & .2317 & & \\
\hline \multirow[t]{3}{*}{60} & 50 & $\beta_{1}$ & -1.0152 & .2014 & $\beta_{2}$ & .9930 & 2091 & -25.68 & 400 \\
\hline & & $\lambda_{1}$ & .4933 & 1939 & $\lambda_{2}$ & 4694 & 2020 & & \\
\hline & & $\sigma_{1}$ & .9241 & .2351 & $\sigma_{2}$ & 1.0125 & 2179 & & \\
\hline \multirow[t]{3}{*}{60} & 100 & $\beta_{1}$ & -1.0146 & .1896 & $\beta_{2}$ & .9975 & .2010 & -25.14 & 399 \\
\hline & & $\lambda_{1}$ & .4837 & 1829 & $\lambda_{2}$ & .4719 & 1796 & & \\
\hline & & $\sigma_{1}$ & .9065 & .2199 & $\sigma_{2}$ & 1.0089 & .2130 & & \\
\hline & 500 & $\overline{\beta_{1}}$ & -1.0130 & .1952 & $\beta_{2}$ & 1.0003 & .1949 & -24.87 & 399 \\
\hline & & $\lambda_{1}$ & .4810 & . 1799 & $\lambda_{2}$ & .4837 & . 1717 & & \\
\hline & & $\sigma_{1}$ & .9158 & .2162 & $\sigma_{2}$ & .9884 & .2248 & & \\
\hline \multirow[t]{3}{*}{60} & 1000 & $\beta_{1}$ & -1.0128 & .1935 & $\beta_{2}$ & 1.0014 & .1935 & -24.77 & 397 \\
\hline & & $\lambda_{1}$ & .4844 & . 1784 & $\lambda_{2}$ & .4821 & . 1756 & & \\
\hline & & $\sigma_{1}$ & .9097 & .2273 & $\sigma_{2}$ & .9840 & .2220 & & \\
\hline \multirow[t]{3}{*}{60} & 3000 & $\beta_{1}$ & -1.0128 & .1926 & $\beta_{2}$ & 1.0028 & .1935 & -24.74 & 399 \\
\hline & & $\lambda_{1}$ & .4826 & . 1805 & $\lambda_{2}$ & .4849 & . 1720 & & \\
\hline & & $\sigma_{1}$ & .9094 & .2263 & $\sigma_{2}$ & .9853 & .2184 & & \\
\hline \multicolumn{10}{|c|}{ Generalized Simulated Pseudo MLE (GSPML) } \\
\hline \multirow[t]{3}{*}{6} & 50 & $\beta_{1}$ & -1.0164 & 1985 & $\beta_{2}$ & .9963 & 2075 & -50.41 & 397 \\
\hline & & $\lambda_{1}$ & .4956 & 1874 & $\lambda_{2}$ & 4808 & 1819 & & \\
\hline & & $\sigma_{1}$ & .9440 & 2018 & $\sigma_{2}$ & 1.0191 & 2176 & & \\
\hline \multirow[t]{3}{*}{60} & 100 & $\beta_{1}$ & -1.0135 & 1896 & $\beta_{2}$ & .9986 & 1941 & -49.11 & 398 \\
\hline & & $\lambda_{1}$ & .4834 & 1837 & $\lambda_{2}$ & .4796 & 1692 & & \\
\hline & & $\sigma_{1}$ & .9234 & 1934 & $\sigma_{2}$ & 1.0082 & 1988 & & \\
\hline \multirow[t]{3}{*}{60} & 500 & $\beta_{1}$ & -1.0122 & 1891 & $\beta_{2}$ & 1.0009 & 1913 & -48.17 & 399 \\
\hline & & $\lambda_{1}$ & .4834 & 1749 & $\lambda_{2}$ & .4874 & 1646 & & \\
\hline & & $\sigma_{1}$ & .9225 & .2029 & $\sigma_{2}$ & .9899 & .2109 & & \\
\hline
\end{tabular}

Notes: CPU time per one replication

SPML: $T=60, \mathrm{~S}=500,28.11$ seconds.

GSPML: $\mathrm{T}=60, \mathrm{~S}=500,177.52$ seconds; 


\section{Concluding Remarks}

Evidence from the Monte Carlo experiment reveals that how discrete states are simulated is important for likelihood simulation. The approach by simulating regime paths based on regime probabilities conditional on current and past sample observations (Hendry and Richard 1992) is superior to other approaches which do not use current sample information. The other approaches suffer from the difficulty of selecting or simulating the proper regime paths in the very large space of regime paths. Comparing the simulated likelihood approach and the simulated pseudo-likelihood approaches, the simulated maximum likelihood estimates are numerically stable and are relatively efficient even in sample with moderate size. For the dynamic switching regression model, the simulated pseudo-likelihood approaches are numerically unstable and their estimates are poor. They can, however, be valuable for the estimation of dynamic disequilibrium models.

This article is in search of computationally tractable and reasonably good simulation approaches for the estimation of general dynamic switching models. While the simulated likelihood approach can be applied to the estimation of such general models, there are statistical issues that have neither been addressed in the article by Hendry and Richard nor in this article. One of the difficulties that has yet to be overcome is to provide a satisfactory procedure for the estimation of standard errors of parameter estimates. As the simulated likelihood approach here has to simulate latent discrete state variables in addition to continuous latent variables, the simulated likelihood function is not smooth. For the simulated pseudo-likelihood approach, Laroque and Salanie (1993) reported the difficulty of constructing adequate estimates of standard errors of their parameter estimators since simulated pseudo-likelihood functions are not differentiable. For the simulated likelihood approach, even though in principle, the information matrix can be used to derive an estimate of the variance of the maximum likelihood estimate, it does not take into account the additional finite sample error due to simulation..$^{9}$ Direct derivatives of the simulated likelihood do not exist as the simulated likelihood function is not differentiable. ${ }^{10}$ Future research on this and related issues on statistical inference is important and remains open.

\footnotetext{
${ }^{9}$ We have tried to simulate the hessian matrix of the exact likelihood function but the resulted variance estimates are quite inadequate. They can underestimate the correct (empirical) standard deviations by more than fifty percent. A similar magnitude of underestimation has been reported for the SPMLE in Laroque and Salanie (1993).

${ }^{10}$ For simulation estimation of discrete choice models, one gets around this problem by simulating probabilities even though choice events are discrete. This is possible, because in a discrete choice model simulation of latent continuous variables by conditioning on observed discrete choices does not require the simulation of latent states. The simulated likelihood approach in Lee (1994) provides a smooth simulated likelihood because it simulates only continuous latent variables. For that approach, the hessian matrix of the simulated likelihood is available and is useful for the construction of estimates for the variance of that simulated likelihood estimator (see Lee 1994 for those results).
} 


\section{References}

Anderson, T.W., 1958, An introduction to multivariate statistical analysis (Wiley, New York, NY).

Borsch-Supan, A. and V.A. Hajivassiliou, 1993, Smooth unbiased multivariate probability simulators for maximum likelihood estimation of limited dependent variable models, Journal of Econometrics 58, $347-368$.

Danielsson, J. and J.F. Richard, 1993, Accelerated gaussian importance sampler with application to dynamic latent variable models, Journal of Applied Econometrics 8, S153-S174.

Cosslett, S.R. and L.F. Lee, 1985, Serial correlation in latent discrete variable models, Journal of Econometrics $27,79-97$.

Fair, R.C. and D.M. Jaffee, 1972, Methods of estimation of markets in disequilibrium, Econometrica 61, $929-952$.

Goldfeld, S.M. and R.E. Quandt, 1973, A markov model for switching regressions, Journal of Econometrics $1,3-15$.

Goldfeld, S.M. and R.E. Quandt, 1975, Estimation in a disequilibrium model and the value of information, Journal of Econometrics 3, 325-348.

Gourieroux, C., A. Monfort and E. Renault, 1983, Indirect inference, Journal of Applied Econometrics 8, S85-S118.

Hajivassiliou, V. and D. McFadden, 1990, The method of simulated scores, with application to models of external debt crises, Cowles Foundation Discussion Paper No. 967 (Cowles Foundation, Yale University).

Hamilton, J.D., 1989, A new approach to the economic analysis of nonstationary time series and the business cycle, Econometrica 57, 357-384.

Hendry, D.F. and J. F. Richard, 1992, Likelihood evaluation for dynamic latent variables models, in: H.M. Amman, D.A. Belsley and L.F. Pau eds., Computational Economics and Econometrics (Kluwer, Amsterdam).

Keane, M.P., 1994, A computationally practical simulation estimator for panel data, Econometrica 62, 95-116.

Kiefer, N.M., 1978, Discrete parameter variation: efficient estimation of a switching regression model, Econometrica 46, 427-434. 


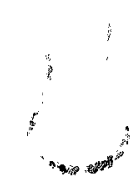

Kim, C.J., 1994, Dynamic linear models with markov-switching, Journal of Econometrics 60: 1-22.

Laroque, G. and B. Salanie, 1993, Simulation-based estimation of models with lagged latent variables, Journal of Applied Econometrics 8, S119-S133.

Laroque, G. and B. Salanie, 1994, Estimating the canonical disequilibrium model: asymptotic theory and finite sample properties, Journal of Econometrics 62, 165-210.

Lee, L.F., 1994, A likelihood simulator for dynamic disequilibrium models, unpublished manuscript (Department of Economics, University of Michigan, Ann Arbor, MI).

Lee, L.F., 1995, Simulation estimation of dynamic switching regression and dynamic disequilibrium models - some monte carlo results, Working paper no. 95-12 (Department of Economics, The Hong Kong University of Science and Technology, Hong Kong).

Lee, L.F. and R.H. Porter, 1984, Switching regression models with imperfect sample separation information, Econometrica 52, 391-418.

Maddala, G.S. and F.D. Nelson, 1974, Maximum likelihood methods for models of markets in disequilibrium, Econometrica 42, 1013-1030.

McFadden, D., 1989, A method of simulated moments of estimation of discrete choice models without numerical integration, Econometrica 57, 995-1026.

Pakes, A. and D. Pollard, 1989, Simulation and the asymptotics of optimization estimators, Econometrica $54,755-785$.

Press, W.H., B.P. Flannery, S.A. Teukolsky, and W.T. Vetterling, 1986, Numerical recipes (Cambridge U. Press, New York).

Quandt, R.E., 1974, A new approach to estimating switching regressions, Journal of the American Statistical Association 67, 306-310.

Quandt, R.E., 1988, The econometrics of disequilibrium (Basil Blackwell, New York, NY).

Quandt, R.E. and J.B. Ramsey, 1978, Estimating mixtures of normal distributions and switching regressions, Journal of the American Statistical Association 73, 730-738.

Shephard, N., 1993, Fitting nonlinear time-series models with applications to stochastic variance models, Journal of Applied Econometrics 8, S135-S152.

Smith, A.A. Jr.; 1993, Estimating nonlinear time-series models using simulated vector autoregressions, Journal of Applied Econometrics 8, S63-S84. 